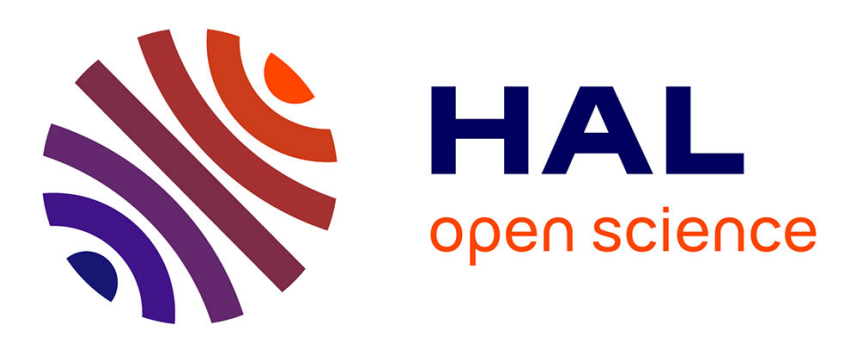

\title{
IIR Youla-Kucera parametrized adaptive feedforward compensators for active vibration control with mechanical coupling
}

\author{
Ioan Doré Landau, Tudor-Bogdan Airimitoaie, Marouane Alma
}

\section{To cite this version:}

Ioan Doré Landau, Tudor-Bogdan Airimitoaie, Marouane Alma. IIR Youla-Kucera parametrized adaptive feedforward compensators for active vibration control with mechanical coupling. IEEE Transactions on Control Systems Technology, 2013, 21 (3), pp.765-779. 10.1109/TCST.2012.2194714 . hal-00673716

\section{HAL Id: hal-00673716 \\ https://hal.science/hal-00673716}

Submitted on 17 Jun 2019

HAL is a multi-disciplinary open access archive for the deposit and dissemination of scientific research documents, whether they are published or not. The documents may come from teaching and research institutions in France or abroad, or from public or private research centers.
L'archive ouverte pluridisciplinaire HAL, est destinée au dépôt et à la diffusion de documents scientifiques de niveau recherche, publiés ou non, émanant des établissements d'enseignement et de recherche français ou étrangers, des laboratoires publics ou privés. 


\title{
IIR Youla-Kucera parameterized adaptive feedforward compensators for active vibration control with mechanical coupling
}

\author{
Ioan Doré Landau, Tudor-Bogdan Airimiţoaie, and Marouane Alma
}

\begin{abstract}
Adaptive feedforward broadband vibration (or noise) compensation requires a reliable correlated measurement with the disturbance (an image of the disturbance). The reliability of this measurement is compromised in most of the systems by a "positive" internal feedback coupling between the compensator system and the correlated measurement of the disturbance. The system may become unstable if the adaptation algorithms do not take into account this positive feedback. Instead of using classical IIR or FIR feedforward compensators, the present paper proposes and analyses an IIR Youla - Kucera parametrization of the feedforward compensator. A model based central IIR stabilizing compensator is used and its performance is enhanced by the adaptation of the parameters (Q-parameters) of an IIR Youla-Kucera filter. Adaptation algorithms assuring the stability of the system in the presence of the positive internal feedback are provided. Their performances are evaluated experimentally on an active vibration control (AVC) system. Theoretical and experimental comparisons with FIR Youla-Kucera parameterized feedforward compensators and IIR feedforward compensators are provided.
\end{abstract}

Index Terms-active vibration control, adaptive feedforward compensation, adaptive control, Youla-Kucera parametrization, parameter estimation.

\section{LIST OF ACRONYMS}

ANC - Active noise control system

AVC - Active vibration control system

FIRYK - Youla-Kucera parameterized IIR adaptive feedforward compensator using a FIR Youla-Kucera filter

IIR - IIR adaptive feedforward compensator

IIRYK - Youla-Kucera parameterized IIR adaptive feedforward compensator using an IIR Youla-Kucera filter

PAA - Parameter adaptation algorithm

PRBS - Pseudo random binary sequence

QFIR - Youla-Kucera FIR filter

QIIR - Youla-Kucera IIR filter

SPR - Strictly positive real (transfer function)

\section{INTRODUCTION}

A preliminary version of this paper has been presented at the CDC/ECC 2011, Orlando, USA. The authors are with the Control System Department of GIPSA-Lab, St. Martin d'Héres, 38402 FRANCE, emails: ([Ioan-Dore.Landau, Tudor-Bogdan.Airimitoaie, Marouane.Alma]@gipsalab.grenoble-inp.fr).

Tudor-Bogdan Airimiţoaie is also with the Faculty of Automatic Control and Computers, University "Politehnica" of Bucharest, Bucharest, 060042 ROMANIA.
DAPTIVE feedforward broadband vibration (or noise) compensation requires a reliable correlated measurement with the disturbance (an image of the disturbance) ([1], [2], [3], [4]). The reliability of this measurement is compromised in most of the systems by a "positive" internal feedback coupling between the compensator system and the correlated measurement of the disturbance. The system may become unstable if the adaptation algorithms do not take into account this positive feedback ([2], [4], [5], [6]). One of the solutions to overcome this problem ([3]) is to try to compensate the positive feedback ([3], [7]). However, since the compensation can not be perfect, the potential instability of the system still exists ([8], [9]).

In the context of this inherent "positive" feedback, the adaptive feedforward compensator should minimize the effect of the disturbance while simultaneously assuring the stability of the internal positive feedback loop.

However this problem can be formulated as a standard feedback control problem using the $2 \times 2$ generalized plant representation [10]. The inputs are the disturbance and the input to the compensator system (the control) and the outputs are the residual acceleration (force, noise) which is the performance variable and the effective measurement of the disturbance. The problem is now to design a feedback compensator (from the measurement of the disturbance to the input of the compensator system) which minimizes the residual acceleration and stabilizes the system ([11], [12]). From a control perspective, the compensator filter appears as a feedback controller while in all the literature dedicated to active vibration (or noise) control the term "feedforward compensator" is used. The term "feedforward" is justified by the fact that the information upon the disturbance is taken "upstream" while for a "feedback compensator" is taken "downstream" by measuring its effect (upon the residual acceleration) ${ }^{1}$.

An approach discussed in the literature is the analysis in this new context of existing algorithms for adaptive feedforward compensation developed for the case without internal coupling. An attempt is made in [8] where the asymptotic convergence in a stochastic environment of the so called "Filtered-U LMS" (FULMS) algorithm is discussed. Further results on the same direction can be found in [7]. The authors use the Ljung's ODE method ([13]) for the case of a scalar vanishing adaptation gain. Unfortunately this is not enough

\footnotetext{
${ }^{1}$ For a coherent presentation with related contributions in the field of active vibration (noise) control, the terminology of the field will be used throughout the paper
} 
because nothing is said about the stability of the system with respect to initial conditions and when a non vanishing adaptation gain is used (to keep adaptation capabilities). The authors assume that the positive feedback does not destabilize the system.

A stability approach for developing appropriate adaptive algorithms in the context of internal positive feedback is discussed in [6] and [14]. Reference [14] provides also an experimental comparison of various algorithms for IIR adaptive compensators in the presence of the internal positive feedback.

In [4], the idea of using an Youla-Kucera parametrization ${ }^{2}$ of the feedforward compensator is illustrated in the context of ANC. Based on the identification of the system, a stabilizing Youla-Kucera controller using an orthonormal basis filter is designed. The Youla-Kucera parameters weighting the orthonormal basis filters are then updated by using a two time scale indirect procedure: (1) estimation of the Qfilter's parameters over a certain horizon, (2) updating of the controller. No stability proof for the tuning procedure is provided.

In [15] an algorithm for adapting the $Q$ parameters of a FIR Youla-Kucera (subsequently called QFIR) parameterized feedforward compensator has been proposed, analyzed and tested experimentally on an AVC system. While the central stabilizing compensator has an IIR structure, the Youla-Kucera filter has a FIR structure.

In the control literature the use of Youla-Kucera type controllers has been extensively discussed. See [16], [17]. Reference [17] gives an extensive coverage of the subject. Related references are also [18], [19] $]^{3}$.

The objectives of this paper are:

- to develop, to analyze, and to evaluate experimentally new recursive algorithms for online estimation and adaptation of the Q-parameters of IIR Youla-Kucera (subsequently called QIIR) parameterized feedforward compensators for broadband disturbances with unknown and variable spectral characteristics;

- to evaluate comparatively these algorithms with respect to existing algorithms from theoretical, implementation, and experimental points of view.

The main contributions of this paper with respect to [4] and [15] are:

- the development of new real time recursive adaptation algorithms for the Q-parameters of IIR Youla-Kucera feedforward compensators and the analysis of the stability of the resulting system;

- the algorithms presented in [15] for FIR Youla-Kucera adaptive feedforward compensators are particular cases of those introduced in this paper;

- application of the algorithms to an AVC system;

- experimental comparison with adaptive IIR feedforward compensators and with adaptive FIR Youla-Kucera parametrization;

\footnotetext{
${ }^{2}$ Throughout the paper the Youla-Kucera parametrization will also be called $Q$ (or $Y K$ ) -parametrization.

${ }^{3}$ To the knowledge of the authors the specific problem considered in this paper is not covered in the existing literature.
}

- significant reduction of the number of parameters to be adapted for the same level of performance when using adaptive IIR Youla-Kucera feedforward compensators instead of adaptive FIR Youla-Kucera feedforward compensators.

In the context of this paper it is assumed that:

- the characteristics of the wide band disturbance acting on the system are unknown and they may vary;

- the internal positive feedback can not be neglected;

- the dynamic models of the AVC are constant and a good estimation of these models is available (these models can be estimated from experimental data).

From the user point of view and taking into account the type of operation of adaptive disturbance compensation systems, one has to consider two modes of operation of the adaptive schemes:

- Adaptive operation. The adaptation is performed continuously with a non vanishing adaptation gain.

- Self-tuning operation. The adaptation procedure starts either on demand or when the performance is unsatisfactory. A vanishing adaptation gain is used.

From an implementation point of view the paper will explore the comparative performances of adaptation algorithms with matrix adaptation gain and with scalar adaptation gain. While the algorithms have been developed and tested in the context of AVC, the results are certainly applicable to ANC systems since they feature the same type of internal positive feedback.

The paper is organized as follows. The AVC system (featuring an internal positive mechanical coupling) on which the algorithms will be tested, is presented in section II. The system representation and the IIR Youla-Kucera feedforward compensator structure are given in section III. The algorithms for adaptive feedforward compensation will be developed in section IV and analyzed in section V. Section VI will present experimental results obtained on the AVC system with the algorithms introduced in this paper as well as an experimental comparison with those given in [14], [15]. Section VII will summarize the comparison with other algorithms.

\section{AN ACTIVE VIBRATION CONTROL SYSTEM USING AN INERTIAL ACTUATOR}

Figures 1 and 2 show an AVC system using a correlated measurement with the disturbance and an inertial actuator for reducing the residual acceleration. The corresponding block diagrams in open loop operation and with the compensator system are shown in Figures 3(a) and 3(b), respectively. The structure is representative for a number of situations encountered in practice (see [12]). It consists on five metal plates (in dural of $1.8 \mathrm{Kg}$ each one) connected by springs. The uppermost and lowermost ones are rigidly jointed together by four screws. The middle three plates will be labeled for easier referencing M1, M2 and M3 (see figure 2). M1 and M3 are equipped with inertial actuators. The one on M1 serves as disturbance generator (inertial actuator I in figure 2), the one at the bottom serves for disturbance compensation (inertial actuator II in figure 2). Inertial actuators use a similar 
principle as loudspeakers (see [20], [21]). The correlated measurement with the disturbance (image of the disturbance) is obtained from an accelerometer which is positioned on plate M1. Another sensor of the same type is positioned on plate M3 and serves for measuring the residual acceleration (see figure 2). The objective is to minimize the residual acceleration measured on plate M3.

When the compensator system is active, the actuator acts upon the residual acceleration, but also upon the measurement of the image of the disturbance through the reverse path (a positive feedback coupling). The measured quantity $\hat{y}(t)$ will be the sum of the correlated disturbance measurement $w(t)$ obtained in the absence of the feedforward compensation (see figure 3(a)) and of the effect of the actuator used for compensation. The disturbance is the position of the mobile part of the inertial actuator (see figures 1 and 2) located on top of the structure. The input to the compensator system is the position of the mobile part of the inertial actuator located on the bottom of the structure. The input to the inertial actuators being a position, the global primary path, the secondary path, and the reverse path have a double differentiator behavior. Similar internal positive feedback coupling occur also in feedforward ANC ([4], [6]).

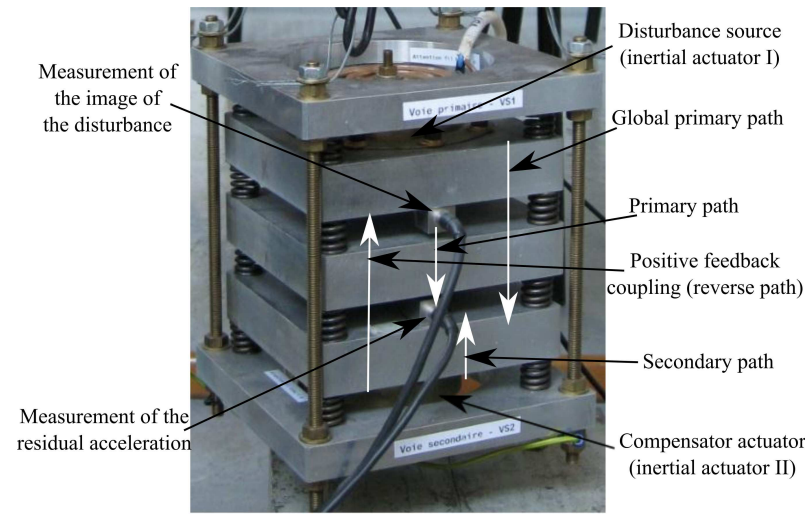

Fig. 1. An AVC system using a feedforward compensation - photo.

In figure $3(\mathrm{~b}), \hat{y}(t)$ denotes the effective output provided by the measurement device and which will serve as input to the adaptive feedforward filter $\hat{N}$. The output of this filter denoted by $\hat{u}(t)$ is applied to the actuator through an amplifier. The transfer function $G$ (the secondary path) characterizes

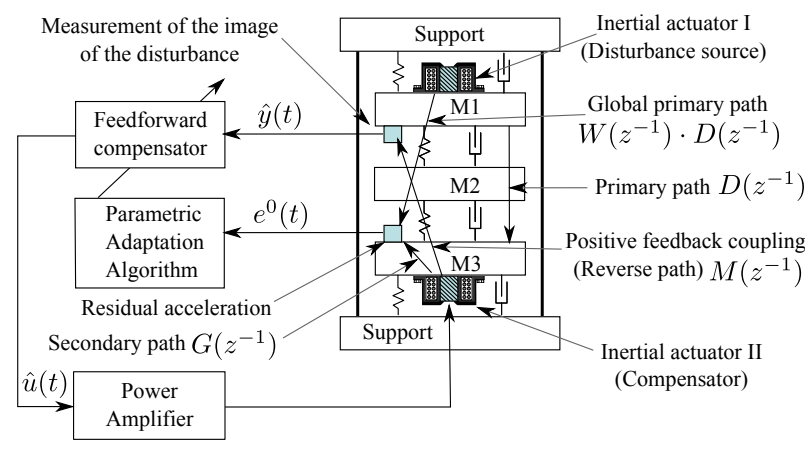

Fig. 2. An AVC system using a feedforward compensation - schema. the dynamics from the output of the filter $\hat{N}$ to the residual acceleration measurement (amplifier + actuator + dynamics of the mechanical system). The transfer function $D$ between $w(t)$ and the measurement of the residual acceleration (in open loop operation) characterizes the primary path.

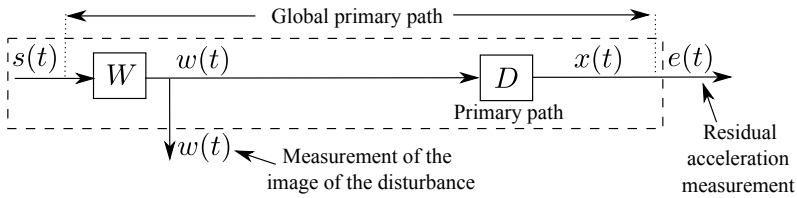

(a)

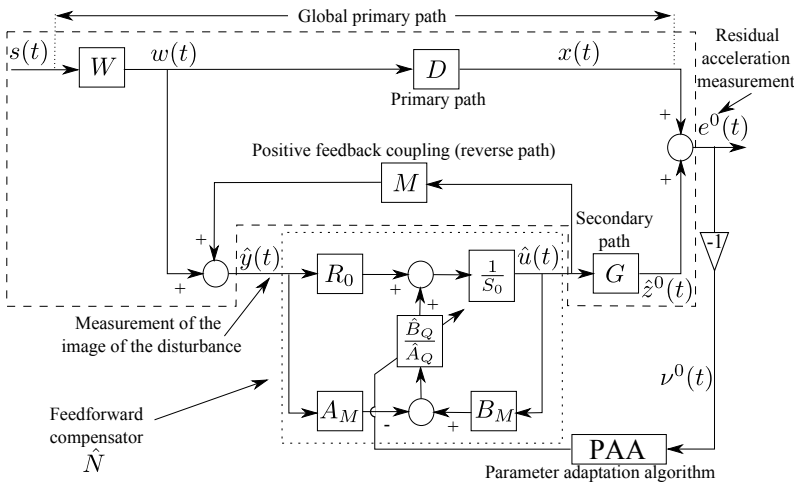

(b)

Fig. 3. Feedforward AVC: in open loop (a) and with adaptive feedforward compensator (b).

The coupling between the output of the filter and the measurement $\hat{y}(t)$ through the compensator actuator is denoted by $M$. As indicated in figure 3(b) this coupling is a "positive" feedback. This unwanted coupling raises problems in practice (source of instabilities) and makes the analysis of adaptive (estimation) algorithms more difficult. The system shown in figure 3(b) can be represented in the standard feedback form shown in Figure 4 (for details see Section III).

At this stage it is important to make the following remarks, when the feedforward filter is absent (open loop operation):

- very reliable models for the secondary path and the "positive" feedback path can be identified by applying appropriate excitation on the actuator used for compensation;

- an initial estimation of the primary path transfer function can be obtained using the measured $w(t)$ as input and $e(t)$ as output (the compensator actuator being at rest);

- the design of a fixed model based stabilizing feedforward compensator requires the knowledge of the reverse path model only;

- the adaptation algorithms do not use information upon the primary path whose characteristics may be unknown or subject to change;

- the knowledge of the disturbance characteristics and of the primary path model in addition of the secondary and reverse paths models is mandatory for the design of an optimal fixed model based feedforward compensator ([11], [12].

The objective is to develop stable recursive algorithms for adaptation of the parameters of the feedforward filter com- 


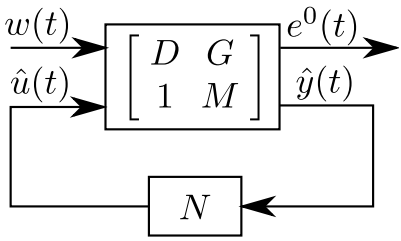

Fig. 4. Feedback representation of the system shown in Figure 3(b).

pensator such that the measured residual error (acceleration or force in AVC, noise in ANC) be minimized in the sense of a certain criterion while simultaneously assuring the stability of the internal positive feedback loop. This has to be done for broadband disturbances $w(t)$ (or $s(t)$ ) with unknown and variable spectral characteristics and an unknown primary path model $^{4}$.

\section{BASIC EQUATIONS AND NOTATIONS}

The block diagrams associated with an AVC system are shown in fig. 3 in open loop (3(a)) and when an IIR (Infinite Impulse Response) Youla-Kucera compensator is active (3(b)).

The primary $(D)$, secondary $(G)$, and reverse (positive coupling) $(M)$ paths represented in fig. 3(b) are characterized by the asymptotically stable transfer operators:

$$
X\left(q^{-1}\right)=\frac{B_{X}\left(q^{-1}\right)}{A_{X}\left(q^{-1}\right)}=\frac{b_{1}^{X} q^{-1}+\ldots+b_{n_{B_{X}}^{X}}^{X} q^{-n_{B_{X}}}}{1+a_{1}^{X} q^{-1}+\ldots+a_{n_{A_{X}}}^{X} q^{-n_{A_{X}}}},
$$

with $B_{X}=q^{-1} B_{X}^{*}$ for any $X \in\{D, G, M\} . \hat{G}=\frac{\hat{B}_{G}}{\hat{A}_{G}}$, $\hat{M}=\frac{\hat{B}_{M}}{\hat{A}_{M}}$, and $\hat{D}=\frac{\hat{B}_{D}}{\hat{A}_{D}}$ denote the identified (estimated) models of $\mathrm{G}, \mathrm{M}$, and $\mathrm{D}$.

The equations associated with the feedback system representation shown in figure 4 are:

$$
\left[\begin{array}{c}
e^{0}(t) \\
\hat{y}(t)
\end{array}\right]=\left[\begin{array}{ll}
P_{11} & P_{12} \\
P_{21} & P_{22}
\end{array}\right]\left[\begin{array}{l}
w(t) \\
\hat{u}(t)
\end{array}\right]=\left[\begin{array}{cc}
D & G \\
1 & M
\end{array}\right]\left[\begin{array}{l}
w(t) \\
\hat{u}(t)
\end{array}\right]
$$

where $e^{0}(t)$ is the performance variable to be minimized (residual acceleration), $\hat{y}(t)$ is the measured variable (image of the disturbance), $w(t)$ is the disturbance $(w(t)=$ $\left.W\left(q^{-1}\right) s(t)\right)$, and $\hat{u}(t)$ is the control input ${ }^{5}$.

The optimal IIR feedforward compensator which will minimize the residual acceleration can be written, using the YoulaKucera parametrization, as

$N\left(q^{-1}\right)=\frac{R\left(q^{-1}\right)}{S\left(q^{-1}\right)}=\frac{A_{Q}\left(q^{-1}\right) R_{0}\left(q^{-1}\right)-B_{Q}\left(q^{-1}\right) A_{M}\left(q^{-1}\right)}{A_{Q}\left(q^{-1}\right) S_{0}\left(q^{-1}\right)-B_{Q}\left(q^{-1}\right) B_{M}\left(q^{-1}\right)}$

where the optimal polynomial $Q\left(q^{-1}\right)$ has an IIR structure

$$
Q\left(q^{-1}\right)=\frac{B_{Q}\left(q^{-1}\right)}{A_{Q}\left(q^{-1}\right)}=\frac{b_{0}^{Q}+b_{1}^{Q} q^{-1}+\ldots+b_{n_{B_{Q}}}^{Q} q^{-n_{B_{Q}}}}{1+a_{1}^{Q} q^{-1}+\ldots+a_{n_{A_{Q}}}^{Q} q^{-n_{A_{Q}}}}
$$

\footnotetext{
${ }^{4}$ Variations of the unknown model $W$, the transfer function between the disturbance $s(t)$ and $w(t)$ are equivalent to variations of the spectral characteristics of $s(t)$.

${ }^{5}$ If $w(t)$ is not measured $P_{21}=0$. If there is no internal positive coupling $M=0$.
}

and $R_{0}\left(q^{-1}\right), S_{0}\left(q^{-1}\right)=1+q^{-1} S_{0}^{*}\left(q^{-1}\right)$ are the polynomials of the central (stabilizing) filter and $A_{M}\left(q^{-1}\right), B_{M}\left(q^{-1}\right)$ are given in $(1)^{6}$.

The estimated $Q$ IIR filter is denoted by $\hat{Q}\left(q^{-1}\right)$ or $\hat{Q}\left(\hat{\theta}, q^{-1}\right)$ when it is a linear filter with constant coefficients or $\hat{Q}\left(t, q^{-1}\right)$ during estimation (adaptation). The vector of parameters of the optimal QIIR filter assuring perfect matching will be denoted by

$$
\theta^{T}=\left[b_{0}^{Q}, \ldots, b_{n_{B_{Q}}}^{Q}, a_{1}^{Q}, \ldots, a_{n_{A_{Q}}^{Q}}^{Q}\right]=\left[\theta_{B_{Q}}^{T}, \theta_{A_{Q}}^{T}\right] .
$$

The vector of parameters for the estimated $Q$ QIIR filter

$$
\hat{Q}\left(q^{-1}\right)=\frac{\hat{B}_{Q}\left(q^{-1}\right)}{\hat{A}_{Q}\left(q^{-1}\right)}=\frac{\hat{b}_{0}^{Q}+\hat{b}_{1}^{Q} q^{-1}+\ldots+\hat{b}_{n_{B_{Q}}}^{Q} q^{-n_{B_{Q}}}}{1+\hat{a}_{1}^{Q} q^{-1}+\ldots+\hat{a}_{n_{A_{Q}}}^{Q} q^{-n_{A_{Q}}}}
$$

is denoted by

$$
\hat{\theta}^{T}=\left[\hat{b}_{0}^{Q}, \ldots, \hat{b}_{n_{B_{Q}}}^{Q}, \hat{a}_{1}^{Q}, \ldots, \hat{a}_{n_{A_{Q}}^{Q}}^{Q}\right]=\left[\hat{\theta}_{B_{Q}}^{T}, \hat{\theta}_{A_{Q}}^{T}\right] .
$$

The input of the feedforward filter (called also reference) is denoted by $\hat{y}(t)$ and it corresponds to the measurement provided by the primary transducer (force or acceleration transducer in AVC or a microphone in ANC). In the absence of the compensation loop (open loop operation) $\hat{y}(t)=w(t)$. The output of the feedforward compensator (which is the control signal applied to the secondary path) is denoted by $\hat{u}(t+1)=\hat{u}(t+1 / \hat{\theta}(t+1))$ (a posteriori output) ${ }^{7}$.

The "a priori" output of the estimated feedforward compensator using an YKIIR parametrization for the case of time varying parameter estimates is given by (using eq. (3))

$$
\begin{aligned}
\hat{u}^{0}(t+1)= & \hat{u}(t+1 / \hat{\theta}(t))=-\hat{S}^{*}\left(t, q^{-1}\right) \hat{u}(t)+\hat{R}\left(t, q^{-1}\right) \hat{y}(t+1) \\
=- & \left(\hat{A}_{Q}\left(t, q^{-1}\right) S_{0}\right)^{*} \hat{u}(t)+\hat{A}_{Q}\left(t, q^{-1}\right) R_{0} \hat{y}(t+1) \\
& +\hat{B}_{Q}\left(t, q^{-1}\right)\left(B_{M}^{*} \hat{u}(t)-A_{M} \hat{y}(t+1)\right)
\end{aligned}
$$

where

$$
\begin{aligned}
\hat{u}(t+1)=- & \left(\hat{A}_{Q}\left(t+1, q^{-1}\right) S_{0}\right)^{*} \hat{u}(t)+\hat{A}_{Q}\left(t+1, q^{-1}\right) R_{0} \hat{y}(t+1) \\
& +\hat{B}_{Q}\left(t+1, q^{-1}\right)\left(B_{M}^{*} \hat{u}(t)-A_{M} \hat{y}(t+1)\right) .
\end{aligned}
$$

It should be observed that eqs. (3), (4), (8), and (9) can be easily particularized for the case of a FIR Youla-Kucera parametrization by taking $\hat{A}_{Q}\left(t, q^{-1}\right) \equiv 1$.

The measured input to the feedforward filter can also be written as

$$
\hat{y}(t+1)=w(t+1)+\frac{B_{M}^{*}\left(q^{-1}\right)}{A_{M}\left(q^{-1}\right)} \hat{u}(t) .
$$

The unmeasurable value of the output of the primary path (when the compensation is active) is denoted $x(t)$. The "a priori" output of the secondary path will be denoted $\hat{z}^{0}(t+$ $1)=\hat{z}(t+1 / \hat{\theta}(t))$ while its input is $\hat{u}(t)$. One has

$$
\hat{z}^{0}(t+1)=\frac{B_{G}^{*}\left(q^{-1}\right)}{A_{G}\left(q^{-1}\right)} \hat{u}(t)=\frac{B_{G}^{*}\left(q^{-1}\right)}{A_{G}\left(q^{-1}\right)} \hat{u}(t / \hat{\theta}(t)),
$$

\footnotetext{
${ }^{6}$ The following notation for polynomials will be used throughout this paper: $A\left(q^{-1}\right)=a_{0}+\sum_{i=1}^{n_{A}} a_{i} q^{-i}=a_{0}+q^{-1} A^{*}\left(q^{-1}\right)$.

${ }^{7}$ In adaptive control and estimation the predicted output at $t+1$ can be computed either on the basis of the previous parameter estimates (a priori) or on the basis of the current parameter estimates (a posteriori).
} 
where $\hat{\theta}(t)$ is the vector of estimated parameters given in (7). The measured residual acceleration (or force) satisfies the following equation

$$
e^{0}(t+1)=x(t+1)+\hat{z}^{0}(t+1) .
$$

The "a priori" adaptation error is defined as

$\nu^{0}(t+1)=\nu(t+1 / \hat{\theta}(t))=-e^{0}(t+1)=-x(t+1)-\hat{z}^{0}(t+1)$.

The "a posteriori" unmeasurable (but computable) adaptation error is given by

$\nu(t+1)=\nu(t+1 / \hat{\theta}(t+1))=-e(t+1)=-x(t+1)-\hat{z}(t+1)$.

where the "a posteriori" value of the output of the secondary path $\hat{z}(t+1)$ (dummy variable) is given by

$$
\hat{z}(t+1)=\hat{z}(t+1 / \hat{\theta}(t+1))=\frac{B_{G}^{*}\left(q^{-1}\right)}{A_{G}\left(q^{-1}\right)} \hat{u}(t / \hat{\theta}(t+1)) .
$$

For compensators with constant parameters $\nu^{0}(t)=\nu(t)$, $e^{0}(t)=e(t), \hat{z}^{0}(t)=\hat{z}(t), \hat{u}^{0}(t)=\hat{u}(t)$.

The objective is to develop stable recursive algorithms for adaptation of the parameters of the $\mathrm{Q}$ filter such that the measured residual error (acceleration or force in AVC, noise in ANC) be minimized in the sense of a certain criterion. This has to be done for broadband disturbances $w(t)$ (or $s(t)$ ) with unknown and variable spectral characteristics and an unknown primary path model.

\section{Development of the Algorithms}

The algorithm for adaptive feedforward YKIIR compensators will be developed under the following hypotheses:

1) H1 - The signal $w(t)$ is bounded (which is equivalently to say that $s(t)$ is bounded and $W\left(q^{-1}\right)$ in figure 3 is asymptotically stable).

2) $\mathrm{H} 2$ - There exists a central feedforward compensator $N_{0}\left(R_{0}, S_{0}\right)$ which stabilizes the inner positive feedback loop formed by $N_{0}$ and $\mathrm{M}$ and the characteristic polynomial of the closed loop ${ }^{8}$

$$
P_{0}\left(z^{-1}\right)=A_{M}\left(z^{-1}\right) S_{0}\left(z^{-1}\right)-B_{M}\left(z^{-1}\right) R_{0}\left(z^{-1}\right)
$$

is a Hurwitz polynomial.

3) H3 - (Perfect matching condition) There exists a value of the $\mathrm{Q}$ parameters such that

$$
\frac{G \cdot A_{M}\left(R_{0} A_{Q}-A_{M} B_{Q}\right)}{A_{Q}\left(A_{M} S_{0}-B_{M} R_{0}\right)}=-D .
$$

4) H4 - The effect of the measurement noise upon the measurement of the residual acceleration is neglected (deterministic context).

Once the algorithm will be developed under these hypotheses, $\mathrm{H} 3$ and $\mathrm{H} 4$ will be removed and the algorithm will be analyzed in this modified context.

A first step in the development of the algorithms is to establish for a fixed estimated compensator a relation between

\footnotetext{
${ }^{8}$ The parenthesis $\left(q^{-1}\right)$ will be omitted in some of the following equations to make them more compact.
}

the error on the Q-parameters (with respect to the optimal values) and the adaptation error $\nu$. This is summarized in the following Lemma.

Lemma 4.1: Under the hypothesis $\mathrm{H} 1$ through $\mathrm{H} 4$ for the system described by equations (1) through (15) using an estimated IIR Youla-Kucera parameterized feedforward compensator with constant parameters one has:

$$
\nu(t+1 / \hat{\theta})=\frac{A_{M}\left(q^{-1}\right) G\left(q^{-1}\right)}{A_{Q}\left(q^{-1}\right) P_{0}\left(q^{-1}\right)}[\theta-\hat{\theta}]^{T} \phi(t),
$$

with $\phi(t)$ given by:

$$
\begin{aligned}
\phi^{T}(t)=\left[\alpha(t+1), \alpha(t), \ldots, \alpha\left(t-n_{B_{Q}}+1\right),\right. & \\
& \left.-\beta(t),-\beta(t-1), \ldots,-\beta\left(t-n_{A_{Q}}\right)\right] .
\end{aligned}
$$

where:

$$
\begin{aligned}
\alpha(t+1) & =B_{M} \hat{u}(t+1)-A_{M} \hat{y}(t+1)= \\
& =B_{M}^{*} \hat{u}(t)-A_{M} \hat{y}(t+1) \\
\beta(t) & =S_{0} \hat{u}(t)-R_{0} \hat{y}(t) .
\end{aligned}
$$

The proof of this lemma is given in Appendix A.

Corollary 4.1: Under the hypothesis $\mathrm{H} 1$ through $\mathrm{H} 4$ for the system described by equations (1) through (15) using an estimated FIR Youla-Kucera parameterized feedforward compensator with constant parameters one has:

$$
\nu(t+1 / \hat{\theta})=\frac{A_{M}\left(q^{-1}\right) G\left(q^{-1}\right)}{P_{0}\left(q^{-1}\right)}[\theta-\hat{\theta}]^{T} \phi(t),
$$

where

$$
\theta^{T}=\left[b_{0}^{Q}, \ldots, b_{n_{B_{Q}}}^{Q}\right]=\left[\theta_{B_{Q}}^{T}\right]
$$

is the vector of parameters of the optimal QFIR filter assuring perfect matching,

$$
\hat{\theta}^{T}=\left[\hat{b}_{0}^{Q}, \ldots, \hat{b}_{n_{B_{Q}}^{Q}}^{Q}\right]=\left[\hat{\theta}_{B_{Q}}^{T}\right]
$$

is the vector of parameters for the estimated $\hat{Q}$ FIR filter

$$
\hat{Q}\left(q^{-1}\right)=\hat{B}_{Q}\left(q^{-1}\right)=\hat{b}_{0}^{Q}+\hat{b}_{1}^{Q} q^{-1}+\ldots+\hat{b}_{n_{B_{Q}}} q^{-n_{B_{Q}}},
$$

and $\phi^{T}(t)$ is given by:

$$
\phi^{T}(t)=\left[\alpha(t+1), \alpha(t), \ldots, \alpha\left(t-n_{B_{Q}}+1\right)\right],
$$

where $\alpha(t+1)$ is given in eq. (19a).

Proof: This result is straightforwardly obtained by making $\hat{A}_{Q}\left(q^{-1}\right)=1$ and $A_{Q}\left(q^{-1}\right)=1$ in Lemma 4.1.

Throughout the remainder of this section and the next one, unless stated differently, the Youla-Kucera parametrization having an QIIR filter will be discussed. It should be observed that in most of the cases results for QFIR-polynomials can be obtained by imposing $A_{Q}\left(q^{-1}\right)=1$ and $\hat{A}_{Q}\left(q^{-1}\right)=1$.

As it will be shown later on, it is convenient for assuring the stability of the system to filter the observation vector $\phi(t)$. Filtering the vector $\phi(t)$ through an asymptotically stable filter $L\left(q^{-1}\right)=\frac{B_{L}}{A_{L}}$, equation (17) for $\hat{\theta}=$ constant becomes

$$
\nu(t+1 / \hat{\theta})=\frac{A_{M}\left(q^{-1}\right) G\left(q^{-1}\right)}{A_{Q}\left(q^{-1}\right) P_{0}\left(q^{-1}\right) L\left(q^{-1}\right)}[\theta-\hat{\theta}]^{T} \phi_{f}(t)
$$


with

$$
\begin{array}{r}
\phi_{f}(t)=L\left(q^{-1}\right) \phi(t)=\left[\alpha_{f}(t+1), \ldots, \alpha_{f}\left(t-n_{B_{Q}}+1\right),\right. \\
\left.\beta_{f}(t), \beta_{f}(t-1), \ldots, \beta_{f}\left(t-n_{A_{Q}}\right)\right]
\end{array}
$$

where

$$
\begin{array}{r}
\alpha_{f}(t+1)=L\left(q^{-1}\right) \alpha(t+1) \\
\beta_{f}(t)=L\left(q^{-1}\right) \beta(t) .
\end{array}
$$

Equation (25) will be used to develop the adaptation algorithms. When the parameters of $\hat{Q}$ evolve over time and neglecting the non-commutativity of the time varying operators (which implies slow adaptation (see [22]), i.e., a limited value for the adaptation gain), equation (25) transforms into 9

$\nu(t+1 / \hat{\theta}(t+1))=\frac{A_{M}\left(q^{-1}\right) G\left(q^{-1}\right)}{A_{Q}\left(q^{-1}\right) P_{0}\left(q^{-1}\right) L\left(q^{-1}\right)}[\theta-\hat{\theta}(t+1)]^{T} \phi_{f}(t)$.

Equation (28) has the standard form for an a-posteriori adaptation error ([23]), which immediately suggests to use the following PAA:

$$
\begin{aligned}
\hat{\theta}(t+1) & =\hat{\theta}(t)+F(t) \psi(t) \nu(t+1) ; \\
\nu(t+1) & =\frac{\nu^{0}(t+1)}{1+\psi^{T}(t) F(t) \psi(t)} ; \\
F(t+1) & =\frac{1}{\lambda_{1}(t)}\left[F(t)-\frac{F(t) \psi(t) \psi^{T}(t) F(t)}{\frac{\lambda_{1}(t)}{\lambda_{2}(t)}+\psi^{T}(t) F(t) \psi(t)}\right] \\
1 & \geq \lambda_{1}(t)>0 ; 0 \leq \lambda_{2}(t)<2 ; F(0)>0 \\
\psi(t) & =\phi_{f}(t),
\end{aligned}
$$

where $\lambda_{1}(t)$ and $\lambda_{2}(t)$ allow to obtain various profiles for the matrix adaptation gain $F(t)$ (see section VI and [23]). By taking $\lambda_{2}(t) \equiv 0$ and $\lambda_{1}(t) \equiv 1$, one gets a constant adaptation gain matrix (and choosing $F=\gamma I, \gamma>0$ one gets a scalar adaptation gain).

Several choices for the filter $L$ will be considered, leading to different algorithms:

$$
\begin{array}{ll}
\text { Algorithm } I & L=G \\
\text { Algorithm IIa } & L=\hat{G} \\
\text { Algorithm } I I b & L=\frac{\hat{A}_{M}}{\hat{P}_{0}} \hat{G} \\
\text { Algorithm III } & \\
& L=\frac{\hat{A}_{M}}{\hat{P}} \hat{G}
\end{array}
$$

with

$$
\hat{P}=\hat{A}_{Q}\left(\hat{A}_{M} S_{0}-\hat{B}_{M} R_{0}\right)=\hat{A}_{Q} \hat{P}_{0},
$$

where $\hat{A}_{Q}$ is an estimation of the denominator of the ideal QIIR filter computed on the basis of available estimates of the parameters of the filter $\hat{Q}$. For the Algorithm $I I I$ several options for updating $\hat{A}_{Q}$ can be considered:

- Run Algorithm $I I a$ or $I I b$ for a certain time to get an estimate of $\hat{A}_{Q}$

- Run a simulation (using the identified models)

- Update $\hat{A}_{Q}$ at each sampling instant or from time to time using Algorithm III (after a short initialization horizon using Algorithm $I I a$ or $I I b$ )

\footnotetext{
${ }^{9}$ However, exact algorithms can be developed taking into account the noncommutativity of the time varying operators - see [23].
}

The following procedure is applied at each sampling time for adaptive or self-tuning operation:

1) Get the measured image of the disturbance $\hat{y}(t+1)$, the measured residual error $e^{0}(t+1)$ and compute $\nu^{0}(t+$ $1)=-e^{0}(t+1)$.

2) Compute $\phi(t)$ and $\phi_{f}(t)$ using (18) and (26).

3) Estimate the parameter vector $\hat{\theta}(t+1)$ using the parametric adaptation algorithm (29a) through (29e).

4) Compute (using (9)) and apply the control.

\section{AnAlysis OF THE Algorithms}

\section{A. The Deterministic Case - Perfect Matching}

For algorithms $I, I I a, I I b$ and $I I I$ the equation for the a-posteriori adaptation error has the form:

$$
\nu(t+1)=H\left(q^{-1}\right)[\theta-\hat{\theta}(t+1)]^{T} \psi(t),
$$

where

$$
H\left(q^{-1}\right)=\frac{A_{M}\left(q^{-1}\right) G\left(q^{-1}\right)}{A_{Q}\left(q^{-1}\right) P_{0}\left(q^{-1}\right) L\left(q^{-1}\right)}, \psi=\phi_{f} .
$$

Neglecting the non-commutativity of time varying operators, one has the following result:

Lemma 5.1: Assuming that eq. (32) represents the evolution of the a posteriori adaptation error when using an IIR YoulaKucera adaptive feedforward compensator and that the PAA (29a) through (29e) is used, one has:

$$
\begin{aligned}
\lim _{t \rightarrow \infty} \nu(t+1) & =0 \\
\lim _{t \rightarrow \infty} \psi(t)[\theta-\hat{\theta}(t+1)] & =0 \\
\lim _{t \rightarrow \infty} \frac{\left[\nu^{0}(t+1)^{2}\right]}{1+\psi(t)^{T} F(t) \psi(t)} & =0 \\
\|\psi(t)\| \text { is bounded } & \\
\lim _{t \rightarrow \infty} \nu^{0}(t+1) & =0
\end{aligned}
$$

for any initial conditions $\hat{\theta}(0), \nu^{0}(0), F(0)$, provided that

$$
H^{\prime}\left(z^{-1}\right)=H\left(z^{-1}\right)-\frac{\lambda_{2}}{2}, \max _{t}\left[\lambda_{2}(t)\right] \leq \lambda_{2}<2
$$

is a SPR transfer function.

The proof of this lemma is given in Appendix B. This result can be particularized for the case of FIR Youla-Kucera adaptive compensators by using the following corollary:

Corollary 5.1: Assuming that eq. (32) represents the evolution of the a posteriori adaptation error for FIR Youla - Kucera adaptive feedforward compensators, where

$$
\begin{aligned}
H\left(q^{-1}\right) & =\frac{A_{M}\left(q^{-1}\right) G\left(q^{-1}\right)}{P_{0}\left(q^{-1}\right) L\left(q^{-1}\right)}, \psi=\phi_{f}, \\
\phi_{f}(t) & =L\left(q^{-1}\right) \phi(t)=\left[\alpha_{f}(t+1), \ldots, \alpha\left({ }_{f} t-n_{B_{Q}}+1\right)\right],
\end{aligned}
$$

and that the PAA (29a) through (29e) is used with $\hat{\theta}(t)$ given by (22), then (34) through (38) hold for any initial conditions $\hat{\theta}(0), \nu^{0}(0), F(0)$, provided that

$$
H^{\prime}\left(z^{-1}\right)=H\left(z^{-1}\right)-\frac{\lambda_{2}}{2}, \max _{t}\left[\lambda_{2}(t)\right] \leq \lambda_{2}<2
$$

is a SPR transfer function. 
The proof is similar to that of Lemma 5.1 and will be omitted.

Remark 1: Using Algorithm III and taking into account eq. (30), the stability condition for $\lambda_{2}=1$ can be transformed into ([13], [24]):

$$
\left|\left(\frac{A_{M}}{\hat{A}_{M}} \cdot \frac{\hat{A}_{Q}}{A_{Q}} \cdot \frac{\hat{P}_{0}}{P_{0}} \cdot \frac{G}{\hat{G}}\right)^{-1}-1\right|<1
$$

for all $\omega$. This roughly means that it always holds provided that the estimates of $A_{M}, A_{Q}, P_{0}$, and $G$ are close to the true values (i.e. $H\left(e^{-j \omega}\right)$ in this case is close to a unit transfer function).

Remark 2: For the case of constant adaptation gain $(F=$ $\alpha I=$ const.) and using Algorithm $I I I$, eq. (29a) can be viewed as an approximation of the gradient algorithm. For constant adaptation gain $\lambda_{2}(t) \equiv 0$ and the strict positive realness on $H^{\prime}\left(z^{-1}\right)$ implies at all the frequencies

$$
-90^{0}<\angle \frac{A_{M}\left(e^{-j \omega}\right) G\left(e^{-j \omega}\right)}{A_{Q}\left(e^{-j \omega}\right) P_{0}\left(e^{-j \omega}\right)}-\angle \frac{\hat{A}_{M}\left(e^{-j \omega}\right) \hat{G}\left(e^{-j \omega}\right)}{\hat{A}_{Q}\left(e^{-j \omega}\right) \hat{P}_{0}\left(e^{-j \omega}\right)}<90^{0} .
$$

Therefore the interpretation of the SPR condition of Lemma 5.1 is that the angle between the direction of adaptation and the direction of the inverse of the true gradient (not computable) should be less than $90^{\circ}$. For time-varying adaptation gains the condition is sharper since in this case $\operatorname{Re}\left\{H\left(e^{-j \omega}\right)\right\}$ should be larger than $\frac{\lambda_{2}}{2}$ at all frequencies.

Remark 3: Eq. (35) indicates that the estimated parameters of the feedforward compensator converge toward the domain $D_{C}=\left\{\hat{\theta}: \psi^{T}(t, \hat{\theta})(\theta-\hat{\theta})=0\right\}$. If furthermore $\psi^{T}(t, \hat{\theta})(\theta-\hat{\theta})=0$ has a unique solution (richness condition), then $\lim _{t \rightarrow \infty} \hat{\theta}(t)=\theta$.

Remark 4: The poles of the estimated $Q$ filter (the roots of $\hat{A}_{Q}$ ), which are also poles of the internal positive closed loop, will be asymptotically inside the unit circle, if the SPR condition is satisfied. However, transiently they may be outside the unit circle. It is possible to force these poles to remain inside of the unit circle during transient using adaptive algorithms with projection (see [23]). However, the SPR condition remains the same.

\section{B. The Stochastic Case - Perfect Matching}

There are two sources of measurement noise, one acting on the primary transducer which gives the correlated measurement with the disturbance and the second acting on the measurement of the residual error (force, acceleration). For the primary transducer the effect of the measurement noise is negligible since the signal to noise ratio is very high. The situation is different for the residual error where the effect of the noise can not be neglected.

In the presence of the measurement noise $(n(t))$, the equation of the a-posteriori residual error becomes

$$
\nu(t+1)=H\left(q^{-1}\right)[\theta-\hat{\theta}(t+1)]^{T} \psi(t)+n(t+1) .
$$

In this context, we should analyze the asymptotic behavior of the adaptation algorithms (i.e., the convergence points in the parameter space). The O.D.E. method [13], [24] can be used to analyse the asymptotic behavior of the algorithm in the presence of noise. Taking into account the form of equation (44), one can directly use Theorem 4.1 of [23] or Theorem B1 of [25].

The following assumptions will be made:

1) $\lambda_{1}(t)=1$ and $\lambda_{2}(t)=\lambda_{2}>0$ (decreasing adaptation gain)

2) $\hat{\theta}(t)$ generated by the algorithm belongs infinitely often to the domain $D_{S}$ :

$$
D_{S} \triangleq\left\{\hat{\theta}: \hat{P}\left(z^{-1}\right)=0 \Rightarrow|z|<1\right\}
$$

for which stationary processes:

$$
\begin{aligned}
\psi(t, \hat{\theta}) & \left.\triangleq \psi(t)\right|_{\hat{\theta}(t)=\hat{\theta}=\text { cons }} \\
e(t, \hat{\theta}) & =\left.e(t)\right|_{\hat{\theta}(t)=\hat{\theta}=\text { const }}
\end{aligned}
$$

can be defined.

3) $n(t)$ is a zero mean stochastic process with finite moments and independent of the sequence $d(t)$.

From (44) for $\hat{\theta}(t)=\hat{\theta}$, one gets

$$
\nu(t+1, \hat{\theta})=H\left(q^{-1}\right)[\theta-\hat{\theta}]^{T} \psi(t, \hat{\theta})+n(t+1) .
$$

Since $\psi(t, \hat{\theta})$ depends upon $w(t)$ only, one concludes that $\psi(t, \hat{\theta})$ and $n(t+1)$ are independent. Therefore using Theorem 4.1 from [23] it results that if

$$
H^{\prime}\left(z^{-1}\right)=\frac{A_{M}\left(z^{-1}\right) G\left(z^{-1}\right)}{A_{Q}\left(z^{-1}\right) P_{0}\left(z^{-1}\right) L\left(z^{-1}\right)}-\frac{\lambda_{2}}{2}
$$

is a SPR transfer function, one has $\operatorname{Prob}\left\{\lim _{t \rightarrow \infty} \hat{\theta}(t) \in D_{C}\right\}=$ 1. If furthermore $\psi^{T}(t, \hat{\theta})(\theta-\hat{\theta})=0$ has a unique solution (richness condition), then $\operatorname{Prob}\left\{\lim _{t \rightarrow \infty} \hat{\theta}(t)=\theta\right\}=1$. Therefore one can say that the parameters of the estimated feedforward compensator will converge to the same value as for the case without noise.

\section{The Case of Non-Perfect Matching}

If $\hat{Q}\left(t, q^{-1}\right)$ does not have the appropriate dimension there is no chance to satisfy the perfect matching condition. Two questions are of interest in this case:

1) The boundedness of the residual error;

2) The bias distribution in the frequency domain.

1) Boundedness of the residual error: For analyzing the boundedness of the residual error, results from [25], [26], can be used. The following assumptions are made:

1) There exists a reduced order filter $\hat{N}$ characterized by the unknown polynomials $\hat{A}_{Q}$ (of order $n_{A_{Q}}$ ) and $\hat{B}_{Q}$ (of order $n_{B_{Q}}$ ) as described in eq. (3), for which the closed loop formed by $\hat{N}$ and $M$ is asymptotically stable, i.e. $\hat{A}_{Q}\left(A_{M} S_{0}-B_{M} R_{0}\right)$ is a Hurwitz polynomial;

2) The output of the optimal filter satisfying the matching condition can be expressed as:

$$
\hat{u}(t+1)=-\left[\hat{S}^{*}\left(q^{-1}\right) \hat{u}(t)-\hat{R}\left(q^{-1}\right) \hat{y}(t+1)+\eta(t+1)\right]
$$

where $\eta(t+1)$ is a norm bounded signal. 
Using the results of [25] (Theorem 4.1 pp. 1505-1506) and assuming that $w(t)$ is norm bounded, it can be shown that all the signals are norm bounded under the passivity condition (39), where $P$ is computed now with the reduced order estimated filter.

2) Bias distribution: Using the Parseval's relation, the asymptotic bias distribution of the estimated parameters in the frequency domain can be obtained starting from the expression of $\nu(t)$, by taking into account that the algorithm minimizes (almost) a criterion of the form $\lim _{N} \frac{1}{N} \sum_{t=1}^{N} \nu^{2}(t)$. Using eq. (16), the bias distribution (for algorithm $I I I$ ) will be given by

$$
\begin{aligned}
\hat{\theta}^{*} & =\arg \min _{\hat{\theta}} \int_{-\pi}^{\pi}\left[\left|D\left(e^{-j \omega}\right)+\frac{\hat{N}\left(e^{-j \omega}\right) G\left(e^{-j \omega}\right)}{1-\hat{N}\left(e^{-j \omega}\right) M\left(e^{-j \omega}\right)}\right|^{2} \phi_{w}(\omega)\right. \\
& \left.+\phi_{n}(\omega)\right] d \omega
\end{aligned}
$$

where $\phi_{w}$ and $\phi_{n}$ are the spectral densities of the disturbance $w(t)$ and of the measurement noise. Taking into account equation (16), one obtains

$$
\begin{aligned}
\hat{\theta}^{*} & =\arg \min _{\hat{\theta}} \int_{-\pi}^{\pi}\left[\left|\frac{G A_{M}^{2}}{P_{0}}\right|^{2}\left|\frac{B_{Q}}{A_{Q}}-\frac{\hat{B}_{Q}}{\hat{A}_{Q}}\right|^{2} \phi_{w}(\omega)\right. \\
& \left.+\phi_{n}(\omega)\right] d \omega .
\end{aligned}
$$

From (49) one concludes that a good approximation of $Q$ filter will be obtained in the frequency region where $\phi_{w}$ is significant and where $G$ has a high gain (usually $G$ should have high gain in the frequency region where $\phi_{w}$ is significant in order to counteract the effect of $w(t)$ ). However the quality of the estimated $\hat{Q}$ filter will be affected also by the transfer function $\frac{A_{M}^{2}}{P_{0}}$.

\section{Relaxing the Positive Real Condition} that:

It is possible to relax the SPR conditions taking into account

1) The disturbance (input to the system) is a broadband signal;

2) Most of the adaptation algorithms work with a low adaptation gain.

Under these two assumptions, the behavior of the algorithm can be well described by the "averaging theory" developed in [22] and [13] (see also [23]).

When using the averaging approach, the basic assumption of a slow adaptation holds for small adaptation gains (constant and scalar in [22] i.e. $\lambda_{2}(t) \equiv 0, \lambda_{1}(t)=1$; matrix and time decreasing asymptotically in [13], [23] i.e $\lim _{t \rightarrow \infty} \lambda_{1}(t)=$ $\left.1, \lambda_{2}(t)=\lambda_{2}>0\right)$

In the context of averaging, the basic condition for stability is that:

$$
\begin{gathered}
\lim _{N \rightarrow \infty} \frac{1}{N} \sum_{t=1}^{N} \psi(t) H^{\prime}\left(q^{-1}\right) \psi^{T}(t)=\frac{1}{2} \int_{-\pi}^{\pi} \Psi\left(e^{j \omega}\right)\left[H^{\prime}\left(e^{j \omega}\right)\right. \\
\left.+H^{\prime}\left(e^{-j \omega}\right)\right] \Psi^{T}\left(e^{-j \omega}\right) d \omega>0
\end{gathered}
$$

be a positive definite matrix $\left(\Psi\left(e^{j \omega}\right)\right.$ is the Fourier transform of $\psi(t)$ ).
One can view (50) as the weighted energy of the observation vector $\psi$. Of course the SPR sufficient condition upon $H^{\prime}\left(z^{-1}\right)$ (see Equation 39) allows to satisfy this condition. However in the averaging context it is only needed that (50) is true which allows that $H^{\prime}$ be non positive real in a limited frequency band. Expression (50) can be re-written as follows ([14]):

$$
\begin{aligned}
& \int_{-\pi}^{\pi} \psi\left(e^{j \omega}\right)\left[H^{\prime}+H^{\prime *}\right] \psi^{T}\left(e^{-j \omega}\right) d \omega= \\
& \sum_{i=1}^{r} \int_{\alpha_{i}}^{\alpha_{i}+\Delta_{i}} \psi\left(e^{j \omega}\right)\left[H^{\prime}+H^{\prime *}\right] \psi^{T}\left(e^{-j \omega}\right) d \omega- \\
& \sum_{j=1}^{p} \int_{\beta_{j}}^{\beta_{j}+\Delta_{j}} \psi\left(e^{j \omega}\right)\left[\bar{H}^{\prime}+\bar{H}^{\prime *}\right] \psi^{T}\left(e^{-j \omega}\right) d \omega>0
\end{aligned}
$$

where $H^{\prime}$ is $\operatorname{SPR}$ in the frequency intervals $\left[\alpha_{i}, \alpha_{i}+\Delta_{i}\right]$ and $\bar{H}^{\prime}=-H^{\prime}$ is positive real in the frequencies intervals $\left[\beta_{j}, \beta_{j}+\Delta_{j}\right]\left(H^{\prime *}\right.$ denotes the complex conjugate of $\left.H^{\prime}\right)$. The conclusion is that $H^{\prime}$ does not need to be SPR. It is enough that the "positive" weighted energy exceeds the "negative" weighted energy. This explains why algorithms $I$, $I I a$ and $I I b$ will work in practice in most of the cases. It is however important to remark that if the disturbance is a single sinusoid (which violates the hypothesis of broadband disturbance) located in the frequency region where $H^{\prime}$ is not SPR, the algorithm may diverge (see [13], [22]). It was observed that despite satisfaction of condition (51) which will assure the stability of the system, attenuation is not very good in the frequency regions where the positive real condition (41) is violated.

Without doubt, the best approach for relaxing the SPR conditions is to use algorithm $I I I$ (given in eq. (30)) instead of algorithm $I I a$ or $I I b$. This is motivated by eq. (42). As it will be shown experimentally, this algorithm gives the best results.

\section{E. Summary of the algorithms}

Table I summarizes the structure of the algorithms and the stability and convergence conditions for the algorithms developed in this paper with matrix and scalar adaptation gain for IIR Youla-Kucera feedforward compensators, for FIR Youla-Kucera feedforward compensators ([15]) and for IIR adaptive feedforward compensators introduced in [14]. These two references take also into account the internal positive feedback. Concerning algorithms for IIR adaptive feedforward compensators, the algorithms introduced in [6] and the FULMS algorithms ([8]) can be viewed as particular cases of those introduced in [14].

It was not possible to give in table I all the options for the adaptation gain. However basic characteristics for adaptive operation (non vanishing adaptation gain) and self-tuning operation (vanishing adaptation gain) have been provided ${ }^{10}$.

\section{EXPERIMENTAL RESULTS}

The detailed description of the system used for the experiments has been given in section II and a photo of the mechanical structure is shown in figure 1 .

\footnotetext{
${ }^{10}$ Convergence analysis can be applied only for vanishing adaptation gains.
} 
TABLE I

COMPARISON OF ALGORITHMS FOR ADAPTIVE FEEDFORWARD COMPENSATION IN AVC WITH MECHANICAL COUPLING

\begin{tabular}{|c|c|c|c|c|c|c|}
\hline & YKIIR & YKFIR & {$[14]$} & YKIIR & YKFIR & {$[14]$} \\
\hline & \multicolumn{3}{|c|}{ Matrix gain } & \multicolumn{3}{|c|}{ Scalar gain } \\
\hline$\hat{\theta}(t+1)=$ & \multicolumn{3}{|c|}{$\hat{\theta}(t)+F(t) \psi(t) \frac{\nu^{0}(t+1)}{1+\psi^{T}(t) F(t) \psi(t)}$} & \multicolumn{3}{|c|}{$\hat{\theta}(t)+\gamma(t) \psi(t) \frac{\nu^{0}(t+1)}{1+\gamma(t) \psi^{T}(t) \psi(t)}$} \\
\hline Adapt. gain & \multirow{2}{*}{\multicolumn{3}{|c|}{$\begin{array}{c}F(t+1)^{-1}=\lambda_{1}(t) F(t)+\lambda_{2}(t) \psi(t) \psi^{T}(t) \\
0 \leq \lambda_{1}(t)<1,0 \leq \lambda_{2}(t)<2, F(0)>0 \\
\text { Decr. gain and const. trace }\end{array}$}} & \multicolumn{3}{|c|}{$\gamma(t)>0$} \\
\hline Adaptive & & & & \multicolumn{3}{|c|}{$\gamma(t)=\gamma=$ const } \\
\hline Self tuning & \multicolumn{3}{|c|}{$\begin{array}{l}\text { Decr. gain and const. trace } \\
\lambda_{2}=\text { const., }, \quad \lim _{t \rightarrow \infty} \lambda_{1}(t)=1\end{array}$} & \multicolumn{3}{|c|}{$\sum_{t=1}^{\infty} \gamma(t)=\infty, \quad \lim _{t \rightarrow \infty} \gamma(t)=0$} \\
\hline$\hat{\theta}(t)=$ & {$\left[\hat{b}_{0}^{Q}, \ldots, \hat{a}_{1}^{Q}, \ldots\right]$} & {$\left[\hat{b}_{0}^{Q}, \ldots\right]$} & {$\left[-\hat{s}_{1}(t), \ldots, \hat{r}_{0}(t)\right.$} & {$\left[\hat{b}_{0}^{Q}, \ldots, \hat{a}_{1}^{Q}, \ldots\right]$} & {$\left[\hat{b}_{0}^{Q}, \ldots\right]$} & {$\left[-\hat{s}_{1}(t), \ldots, \hat{r}_{0}(t),\right.$.} \\
\hline$\phi^{T}(t)=$ & $\begin{array}{c}{[\alpha(t+1), \ldots, \beta(t), \ldots]} \\
\alpha(t)=B_{M} \hat{u}(t)-A_{M} \hat{y}(t \\
\beta(t)=R_{0} \hat{y}(t)-S_{0} \hat{u}(t)\end{array}$ & $\left\{\begin{array}{c}\alpha(t+1), \ldots] \\
\alpha(t)=B_{M} \hat{u}(t) \\
-A_{M} \hat{y}(t)\end{array}\right.$ & $\begin{array}{c}{[-\hat{u}(t), \ldots} \\
\hat{y}(t+1), \ldots]\end{array}$ & $\begin{array}{c}\alpha \alpha(t+1), \ldots, \beta(t), \ldots] \\
\alpha(t)=B_{M} \hat{u}(t)-A_{M} \hat{y}(t) \\
\beta(t)=R_{0} \hat{y}(t)-S_{0} \hat{u}(t)\end{array}$ & $\mid \begin{array}{c}\alpha(t+1), \ldots] \\
\alpha(t)=B_{M} \hat{u}(t) \\
-A_{M} \hat{y}(t)\end{array}$ & $\begin{array}{l}{[-\hat{u}(t), \ldots,} \\
\hat{y}(t+1), \ldots]\end{array}$ \\
\hline $\begin{array}{l}\hat{P}= \\
P=\end{array}$ & $\begin{array}{l}\hat{A}_{Q}\left(\hat{A}_{M} S_{0}-\hat{B}_{M} R_{0}\right) \\
A_{Q}\left(A_{M} S_{0}-B_{M} R_{0}\right)\end{array}$ & \begin{tabular}{|c|}
$\hat{A}_{M} S_{0}-\hat{B}_{M} R_{0}$ \\
$A_{M} S_{0}-B_{M} R_{0}$
\end{tabular} & $\begin{array}{l}\hat{A}_{M} \hat{S}-\hat{B}_{M} \hat{R} \\
A_{M} \hat{S}-B_{M} \hat{R}\end{array}$ & $\begin{array}{l}\hat{A}_{Q}\left(\hat{A}_{M} S_{0}-\hat{B}_{M} R_{0}\right) \\
A_{Q}\left(A_{M} S_{0}-B_{M} R_{0}\right)\end{array}$ & $\mid$\begin{tabular}{|c|}
$\hat{A}_{M} S_{0}-\hat{B}_{M} R_{0}$ \\
$A_{M} S_{0}-B_{M} R_{0}$
\end{tabular} & \begin{tabular}{|l}
$\hat{A}_{M} \hat{S}-\hat{B}_{M} \hat{R}$ \\
$A_{M} \hat{S}-B_{M} \hat{R}$
\end{tabular} \\
\hline$\psi(t)=$ & \multicolumn{3}{|c|}{$L \phi(t) ; \quad L_{2}=\hat{G} ; \quad L_{3}=\frac{\hat{A}_{M}}{\hat{P}} \hat{G}$} & \multicolumn{3}{|c|}{$L \phi(t) ; \quad L_{2}=\hat{G} ; \quad L_{3}=\frac{\hat{A}_{M}}{\hat{P}} \hat{G}$} \\
\hline $\begin{array}{l}\text { Stability } \\
\text { condition }\end{array}$ & \multicolumn{3}{|c|}{$\frac{A_{M} G}{P L}-\frac{\lambda}{2}=S P R \quad\left(\lambda=\max \lambda_{2}(t)\right)$} & \multicolumn{3}{|c|}{$\frac{A_{M} G}{P L}=S P R$} \\
\hline $\begin{array}{c}\text { Conv. } \\
\text { condition }\end{array}$ & \multicolumn{3}{|c|}{$\frac{A_{M} G}{P L}-\frac{\lambda}{2}=S P R \quad\left(\lambda=\lambda_{2}\right)$} & \multicolumn{3}{|c|}{$\frac{A_{M} G}{P L}=S P R$} \\
\hline
\end{tabular}

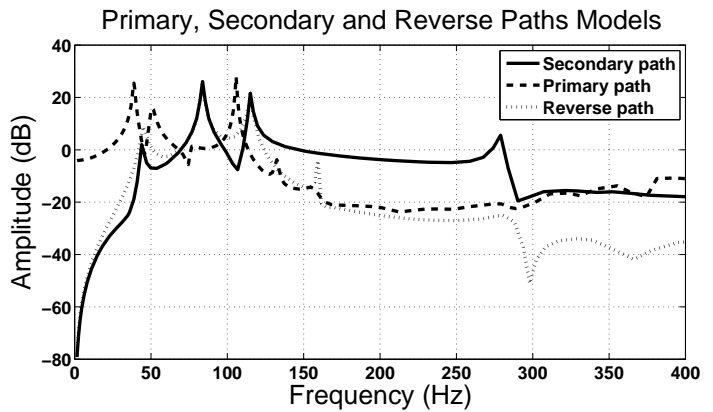

Fig. 5. Frequency characteristics of the primary, secondary and reverse paths

\section{A. System identification}

The methodology used for parametric identification of the mechanical structure's paths is similar to that of [14], [26], [27]. The sampling frequency is $800 \mathrm{~Hz}$.

The secondary and reverse paths have been identified in the absence of the feedforward compensator (see figure 3(b)) using as excitation signal a PRBS generated by a 10 bit shift register and a frequency divider $p=4$ applied at the input of the amplifier feeding the inertial actuator used for compensation ${ }^{11}$ (see figures 1 and 2). For the secondary path, $G\left(q^{-1}\right)$, the output is the residual acceleration measurement, $e(t)$. For the reverse path, $M\left(q^{-1}\right)$, the output is the signal delivered by the primary transducer (accelerometer) $\hat{y}(t)$.

The estimated orders of the model for the secondary path are $n_{B_{G}}=14, n_{A_{G}}=14$. The best results, in terms of validation, have been obtained with the Recursive Extended Least Square method. The frequency characteristic of the secondary path is shown in figure 5, solid line. It features several very low damped vibration modes. The first vibration mode is at $44 \mathrm{~Hz}$

\footnotetext{
${ }^{11}$ It was first verified with $p=2$ that there are no significant dynamics around $200 \mathrm{~Hz}$ and then $p=4$ has been chosen in order to enhance the power spectral density of the excitation in low frequencies while keeping a reasonable length for the experiment.
}

with a damping of 0.0212 , the second at $83.8 \mathrm{~Hz}$ with a damping of 0.00961 , the third one at $115 \mathrm{~Hz}$ with a damping of 0.00694 . There is also a pair of low damped complex zeros at $108 \mathrm{~Hz}$ with a damping of 0.021 . As a consequence of the double differentiator behavior, a double zero at $z=1$ is also present.

For the reverse path $M\left(q^{-1}\right)$, the model's complexity has been estimated to be $n_{B_{M}}=13, n_{A_{M}}=13$. The frequency characteristic of the reverse path is shown in figure 5 (dotted line). There are several very low damped vibration modes at $45.1 \mathrm{~Hz}$ with a damping of 0.0331 , at $83.6 \mathrm{~Hz}$ with a damping of 0.00967 , at $115 \mathrm{~Hz}$ with a damping of 0.0107 and some additional modes in high frequencies. There are two zeros on the unit circle corresponding to the double differentiator behavior. The gain of the reverse path is of the same order of magnitude as the gain of the secondary path up to $150 \mathrm{HZ}$, indicating a strong feedback in this frequency zone.

The primary path has been identified in the absence of the feedforward compensator using $w(t)$ as an input and measuring $e(t)$. The disturbance $s(t)$ was a PRBS sequence $(\mathrm{N}=10$, frequency divider $\mathrm{p}=2)$. The estimated orders of the model are $n_{B_{D}}=26, n_{A_{D}}=26$. The frequency characteristic is presented in figure 5 (dashed line) and may serve for simulations and detailed performance evaluation. Note that the primary path features a strong resonance at $108 \mathrm{~Hz}$, exactly where the secondary path has a pair of low damped complex zeros (almost no gain). Therefore one can not expect good attenuation around this frequency.

\section{B. The central controllers and comparison objectives}

Two central controllers have been used to test IIRYK adaptive feedforward compensators. The first (PP) has been designed using a pole placement method adapted for the case of positive feedback systems. Its main objective is to stabilize the internal positive feedback loop. The end result was a controller of orders $n_{R_{0}}=15$ and $n_{S_{0}}=17$. The second 
$\left(H_{\infty}\right)$ is a reduced order $H_{\infty}$ controller with $n_{R_{0}}=19$ and $n_{S_{0}}=20$ from $[11]^{12}$. For the design of the $H_{\infty}$ controller, the knowledge of the primary path is mandatory (which is not necessary for the PP controller). Figure 6 shows a comparison of the performances obtained with these controllers. One observes that $H_{\infty}$ already provides a good attenuation $(14.70 \mathrm{~dB})^{13}$.

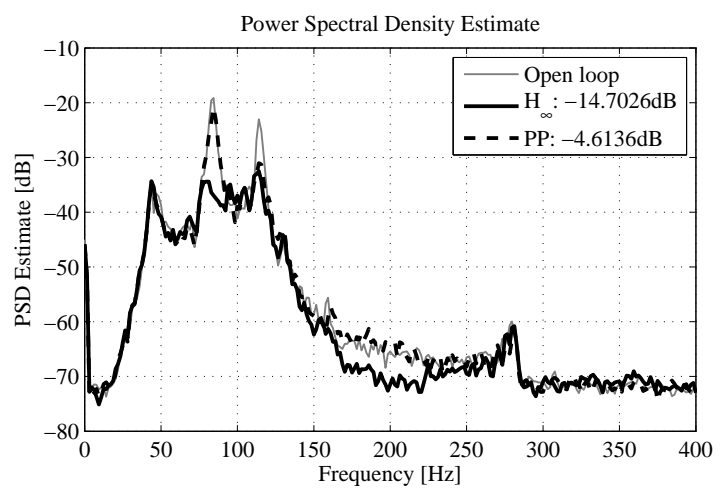

Fig. 6. Spectral densities of residual acceleration for the two central controllers (experimental)

\section{Broadband disturbance rejection using matrix adaptation gain}

Broadband disturbance rejection capabilities using the two Youla-Kucera parametrizations with IIR and FIR filters described in column 2 and 3 of table I are evaluated in this subsection and some observations regarding how they compare to the algorithm of column 4 (see also [14]) are made. For most of the experiments, the complexity of the IIRYK filter was $n_{B_{Q}}=3$ and $n_{A_{Q}}=8$, therefore having 12 parameters in the adaptation algorithm according to eq. (4). For the FIRYK parametrization, an adaptive filter of order $n_{Q}=31$ (32 parameters) has been used. These values do not allow for the "perfect matching condition" to be verified.

A PRBS excitation on the global primary path is considered as the disturbance.

Two modes of operation can be considered, depending on the particular choices taken in eq. (29c):

- For adaptive operation, Algorithms $I I a$ and $I I I$ have been used with decreasing adaptation gain $\left(\lambda_{1}(t)=1\right.$, $\left.\lambda_{2}(t)=1\right)$ combined with a constant trace adaptation gain. When the trace of the adaptation matrix is bellow a given value, the constant trace gain updating modifies the values of $\lambda_{1}(t)$ and $\lambda_{2}(t)$ so that the trace of $F$ is kept constant. This assures the evolution of the PAA in the optimal direction but the step size does not go to zero, therefore maintaining adaptation capabilities for eventual changes in disturbance or variations of the primary path model.

- In self-tuning operation, a decreasing adaptation gain $F(t)$ is used and the step size goes to zero. Then, if

\footnotetext{
${ }^{12}$ The orders of the initial $H_{\infty}$ controller were: $n_{R_{H_{\infty}}}=70$ and $n_{S_{H_{\infty}}}=70$.

${ }^{13}$ The same central controllers have been used in [15] for evaluating FIRYK feedforward adaptive compensators.
}

a degradation of the performance is observed, as a consequence of a change of the disturbance characteristics, the PAA is re-started.

The parametric adaptation algorithms have been implemented using the UD factorization $[23]^{14}$. For reason of space only the experimental results in adaptive operation will be presented. For IIRYK the adaptation has been done starting with an initial gain of 0.02 (initial trace $=$ initial gain $\times$ number of adjustable parameters, thus 0.24) and using a constant trace of 0.02 . For FIRYK an initial gain of 0.05 (initial trace $0.05 \times 32=1.6)$ and constant trace 0.1 have been used.

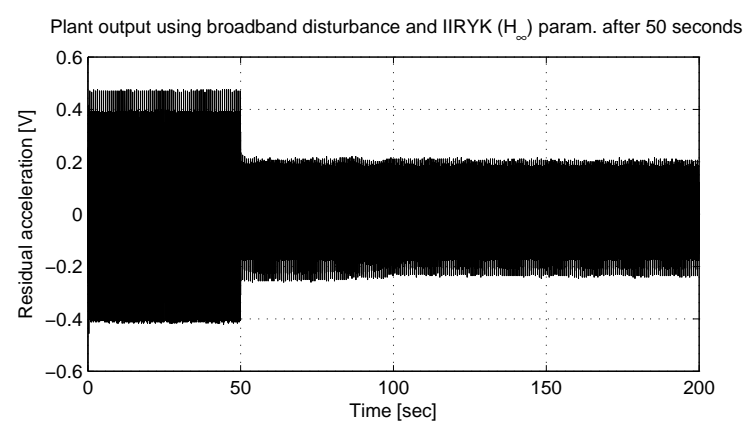

Fig. 7. Real time residual acceleration obtained with the IIR Youla-Kucera parametrization $\left(n_{B_{Q}}=3, n_{A_{Q}}=8\right)$ using Algorithm IIa with matrix adaptation gain and the $H_{\infty}$ central controller.

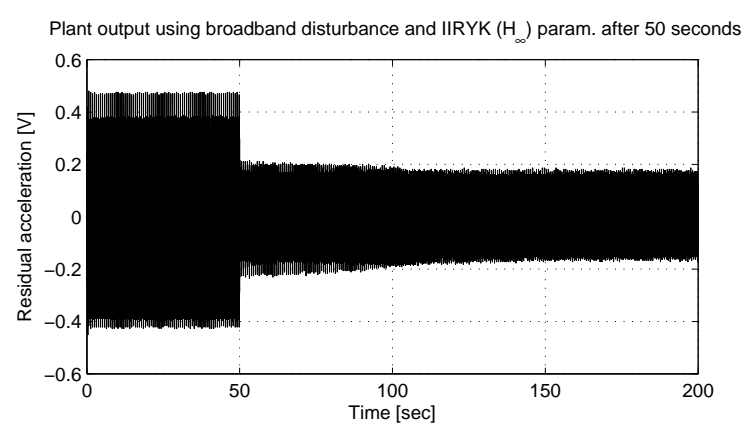

Fig. 8. Real time residual acceleration obtained with the IIR Youla-Kucera parametrization $\left(n_{B_{Q}}=3, n_{A_{Q}}=8\right)$ using Algorithm III with matrix adaptation gain and the $H_{\infty}$ central controller.

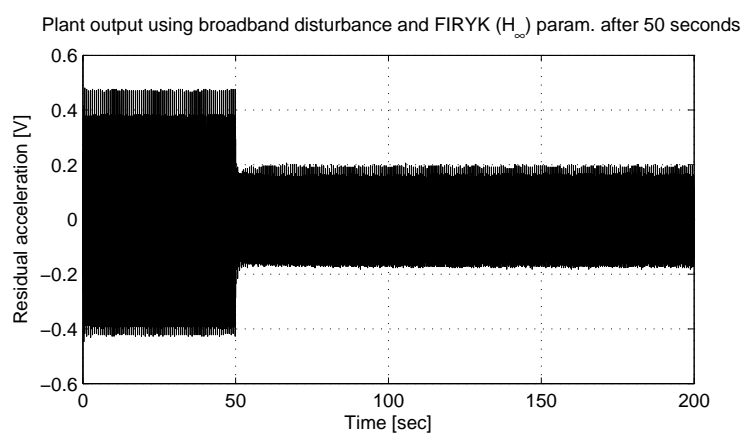

Fig. 9. Real time results obtained with the FIR Youla-Kucera parametrization $\left(n_{Q}=31\right)$ using Algorithm $I I I$ with matrix adaptation gain and the $H_{\infty}$ central controller.

\footnotetext{
${ }^{14}$ An array implementation as in [28] can be also considered.
} 
The experiments have been carried out by first applying the disturbance and then starting the adaptive feedforward compensation after 50 seconds using the FIR or the IIR YoulaKucera parametrization. If not otherwise specified, the results which will be presented have been obtained with the $H_{\infty}$ central controller. In the case of the IIRYK parametrization using Algorithm $I I I$, the filtering by the denominator of the QIIR filter used in equation (31) is done adaptively by using the last stable estimation of $A_{Q}\left(q^{-1}\right)$. Time domain results using IIRYK with Algorithms $I I a$ and $I I I$ are shown in figures 7 and 8 respectively. It can be seen that Algorithm III provides a better performance than Algorithm II $a$ and this can be explained by a better approximation of the positive real condition (see discussion in subsection V-D). Figure 9 shows the evolution of the residual acceleration with the FIRYK adaptive compensator using Algorithm III of [15]. The final attenuation given by IIRYK using Algorithm III (16.21dB) is better than that provided by IIRYK using Algorithm II a $(13.37 d B)$ and slightly better than that provided by using FIRYK with Algorithm III (16.17dB) which uses significantly more adjustable parameters (32 instead of 12). However the adaptation transient is slightly more rapid for FIRYK.

The power spectral density of the residual acceleration (after adaptation transient is finished) for the considered algorithms are shown in fig. 10.

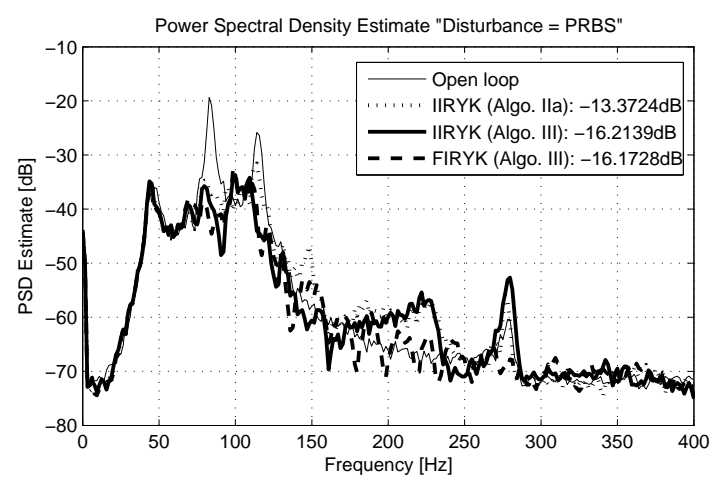

Fig. 10. Power spectral densities of the residual acceleration in open loop, with IIRYK $\left(n_{B_{Q}}=3, n_{A_{Q}}=8\right)$ and with FIRYK $\left(n_{Q}=31\right)$ using the $H_{\infty}$ central controller (experimental).

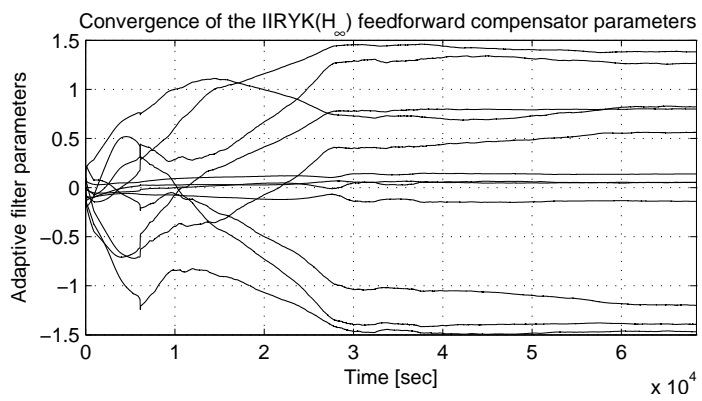

Fig. 11. Evolution of the IIRYK parameters $\left(n_{B_{Q}}=3, n_{A_{Q}}=8\right.$ and $H_{\infty}$ central controller) for Algorithm III using matrix adaptation gain (experimental).

Figure 11 shows the convergence of the parameters for the IIRYK feedforward adaptive compensator using Algorithm
III. The experiment has been carried out over an horizon of 13 hours. Parameters take approximatively 8 hours to almost settle. However this does not affect the performance (the transient duration on the residual acceleration for Algorithm III is about $50 \mathrm{~s}$ ).

An evaluation of the influence of the number of parameters upon the global attenuation of the IIRYK parametrization is shown in table II. The results are grouped on two lines corresponding to the two central controllers used, and the given attenuations are measured in $d B$. The column headers give the number of numerator coefficients followed by the number of denominator coefficients. It can be observed that a larger order of the denominator is better than a larger order of the numerator.

\begin{tabular}{|c|c|c|c|c|c|}
\hline Total no. param. & 0 & 8 & 12 & & 16 \\
\hline No. param. of num/den & $0 / 0$ & $4 / 4$ & $4 / 8$ & $6 / 6$ & \begin{tabular}{|l|l|l|}
$10 / 6$ & $6 / 10$ & $8 / 8$
\end{tabular} \\
\hline$H_{\infty}(\mathrm{db})$ & 14.7 & 15.96 & 15.5616 .21 & 16.31 & \begin{tabular}{|l|l|l|}
15.67 & 16.5 & 16.47
\end{tabular} \\
\hline PP (db) & 4.61 & 15.52 & \begin{tabular}{|l|l|}
16.25 & 16.02 \\
\end{tabular} & 16.24 & $15.57 \mid 15.7216 .21$ \\
\hline
\end{tabular}

INFLUENCE OF THE NUMBER OF THE IIRYK PARAMETERS UPON THE GLOBAL ATTENUATION

A similar analysis for the FIRYK feedforward adaptive compensators is given in table III. Comparing the two tables one can say that a reduction of adjustable parameters by a factor of (at least) 2 is obtained in the case of IIRYK with respect to to FIRYK for approximatively same level of performance (compare IIRYK with 8 parameters with the FIRYK with 16 and the IIRYK with $6 / 6$ parameters with the FIRYK with 32 parameters). It can be noticed that the IIRYK is less sensitive that FIRYK with respsect to the performances of the model based central controller. Table III gives also comparative results for the IIR adaptive fedforward compensators. The IIRYK structure seems to allows a slight reduction of the number of parameters with respect to the IIR structure for the same level of performance (compare the results of IIRYK with 16 adjustable parameters (6/10) with the IIR using 20 adjustable parameters).

\begin{tabular}{|c|c|c|c|c|c|c|}
\hline No. param. & 0 & 8 & 16 & 20 & 32 & 40 \\
\hline$H_{\infty}(\mathrm{db})$ & 14.7 & 15.4 & 15.6 & - & 16.17 & 16.03 \\
\hline PP $(\mathrm{db})$ & 4.61 & 14.69 & 15.89 & - & 15.7 & 15.33 \\
\hline IIR $(\mathrm{db})$ & & - & - & 16.23 & 16.49 & 16.89 \\
\hline
\end{tabular}

TABLE III

INFLUENCE OF THE NUMBER OF PARAMETERS UPON THE GLOBAL ATTENUATION FOR THE FIRYK PARAMETRIZATION (LINES 2 AND 3) AND FOR THE IIR ADAPTIVE FILTER (LINE 4)

To verify the adaptive capabilities of the two parametrizations, a narrow band disturbance has been added after 1400 seconds of experimentation. This has been realized by using a sinusoidal signal of $150 \mathrm{~Hz}$. Power spectral density estimates are shown in fig. 12 for the IIRYK parametrization and in fig. 13 for the FIRYK parametrization. Better results are obtained with the IIRYK parametrization and they are comparable with those obtained for IIR adaptive feedforward compensators. See [14, Fig. 12]. 


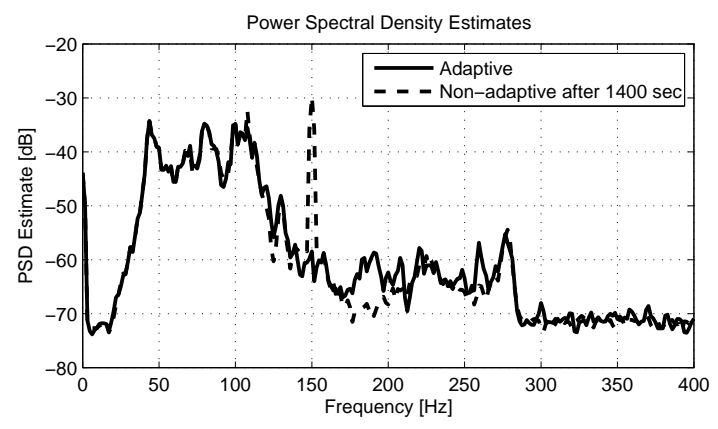

Fig. 12. Power spectral densities of the residual acceleration when an additional sinusoidal disturbance is added (Disturbance $=$ PRBS + sinusoid) and the IIRYK parametrization is used.

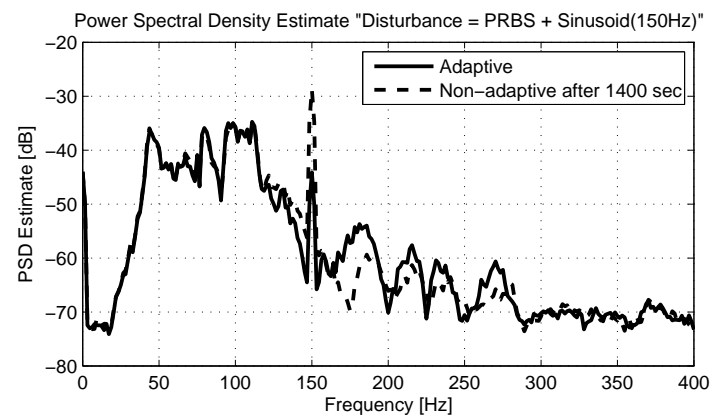

Fig. 13. Power spectral densities of the residual acceleration when an additional sinusoidal disturbance is added (Disturbance $=$ PRBS + sinusoid) and the FIRYK parametrization is used.

\section{Broadband disturbance rejection using scalar adaptation gain}

The scalar adaptation gain algorithms of columns 5 and 6 from table I have been also tested on the AVC system.

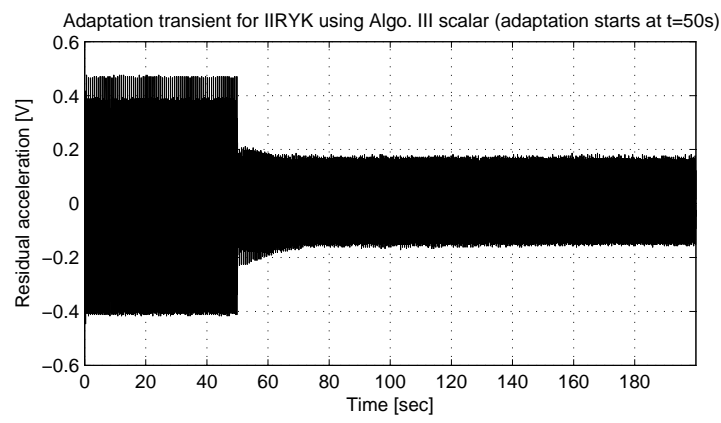

Fig. 14. Real time residual acceleration obtained with the IIR Youla-Kucera parametrization $\left(n_{B_{Q}}=3, n_{A_{Q}}=8\right)$ using Algorithm III with scalar adaptation gain and the $H_{\infty}$ central controller.

In the adaptation regime, as opposed to the matrix cases, a constant adaptation gain of 0.001 has been used for both parametrizations, as in [14] (see also table I). This corresponds to a constant trace of 0.012 for the IIRYK and 0.032 for the FIRYK (taking into account the number of adapted parameters). Figure 14 shows the adaptation transient for the scalar version of the IIRYK parametrization using Algorithm III. Surprisingly, the performances are close to those obtained with a matrix adaptation gain. (a similar observation has been made in [14, Fig. 14]. Figure 15 shows the adaptation

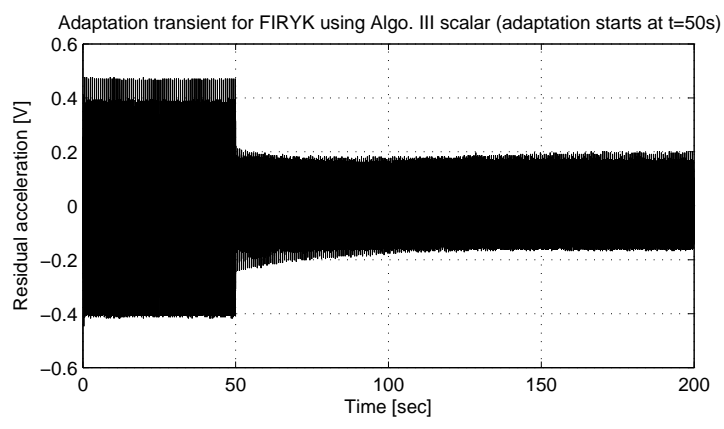

Fig. 15. Real time residual acceleration obtained with the FIR Youla-Kucera parametrization $\left(n_{Q}=31\right)$ using Algorithm $I I I$ with scalar adaptation gain and the $H_{\infty}$ central controller.

transient for the FIRYK parametrization using a scalar adaptation gain. It can be seen that the transient performances are a little better for the IIRYK. In fig. 16, power spectral densities and the corresponding global attenuations are given for both parametrizations. It can be observed that IIRYK parametrization with 12 adjustable parameters gives a slightly better attenuation (additional $0.5 \mathrm{~dB}$ ) with respect to a FIRYK parametrization with 32 parameters.

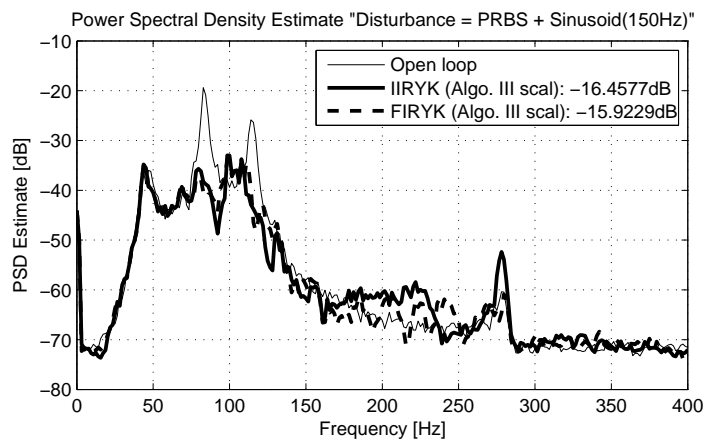

Fig. 16. Power spectral densities of the residual acceleration in open loop, with IIRYK $\left(n_{B_{Q}}=3, n_{A_{Q}}=8\right)$ and with FIRYK $\left(n_{Q}=31\right)$ using scalar adaptation gain and the $H_{\infty}$ central controller (experimental).

\section{COMPARISON WITH OTHER ALGORITHMS}

The algorithms developed in this paper with matrix and scalar adaptation gains for IIR Youla-Kucera feedforward compensators have been compared with the FIR Youla-Kucera parameterized feedforward compensators from [15] and the direct IIR adaptive algorithm of [14] (see Table I). This section summarizes the observations made in Subsection V-E and in Section VI based on experimental results.

Remark 1 - The number of adjustable parameters. The main advantage of the IIRYK adaptive feedforward compensators introduced in this paper compared with FIRYK adaptive compensators is that they require a significantly lower number of adjustable parameters for a given level of performance (a reduction by a factor of 2 in the application presented). This is without doubt a major practical advantage in terms of implementation complexity. A slight reduction of the number of adjustable parameters is also obtained with respect to IIR adaptive feedforward compensators. 
Remark 2 - The poles of the internal positive closed loop. For IIR adaptive feedforward compensators provided that the SPR condition for stability is satisfied, the poles of the internal "positive" loop will be asymptotically stable but they can be very close to the unit circle. For FIRYK, the poles of the internal positive feedback loop are assigned by the central stabilizing controller and they remain unchanged under the effect of adaptation. For IIRYK, part of the poles of the internal positive feedback loop are assigned by the central stabilizing controller but there are additional poles corresponding to $\hat{A}_{Q}$. These poles will be inside the unit circle if the positive real condition for stability is satisfied but they can be very close to the unit circle (at least theoretically). However if one likes to impose that these poles lie inside a circle of a certain radius, this can be easily achieved by using parameter adaptation algorithms with "projections" ([23], [29]).

Remark 3 - Implementation of the filter for Algorithm III. For IIRYK adaptive compensator one has to run first algorithm $I I a$ or $I I b$ over a short horizon in order to get an estimate of $\hat{A}_{Q}$ for implementing the appropriate filter. A similar procedure has to be used also for IIR adaptive compensators (See [14]). For the IIRYK the filter can be continuously improved by updating at each step the estimation of $\hat{A}_{Q}$ in the filter. Such a procedure is more difficult to apply to the IIR structure since the estimated closed loop poles have to be computed at each step based on current estimates of the feedforward compensator's parameters and the knowledge of the reverse path $M\left(q^{-1}\right)$. For FIRYK this initialization procedure is not necessary since the poles of the internal positive feedback loop remain unchanged under the effect of adaptation and a good estimation is provided by the knowledge of the central stabilizing compensator and of the model of the reverse path.

Remark 4 - Initial model based design compensator. Since the system as well as the initial characteristics of the disturbance can be identified, a model based design of an initial feedforward compensator can be done. For a FIRYK or an IIRYK adaptive feedforward compensator, any model based designed compensator can be used as the central controller (no matter what is its dimension). Its performances will be enhanced by the adaptation of the Q-parameters. However, for IIR adaptive feedforward compensators the initial model based designed compensator should have the same structure (number of parameters) as the adaptive structure.

Remark 5 - Influence of the initial stabilizing controller. The performances of IIRYK adaptive compensator are less sensitive that those of FIRYK adaptive compensator with respect to the performances of the initial model based stabilizing controller (at least for a reduced number of adjustable parameters).

\section{CONCLUDing REMARKS}

The paper has presented an adaptive IIR Youla-Kucera parameterized feedforward compensator built around a stabilizing filter for the internal "positive" feedback loop occurring in AVC and ANC systems. Experimental results on an AVC system featuring an internal "positive" feedback have illustrated the potential of the approach. It has been shown that the use of the IIR Youla-Kucera filters allows to reduce significantly the number of parameters to be adapted with respect to the FIR Youla-Kucera filters for the same level of performance.

\section{APPENDIX A}

PROOF OF LEMMA 4.1

Proof: Using hypothesis $\mathrm{H} 3$, one can construct an equivalent closed loop system for the primary path as in figure 17 .

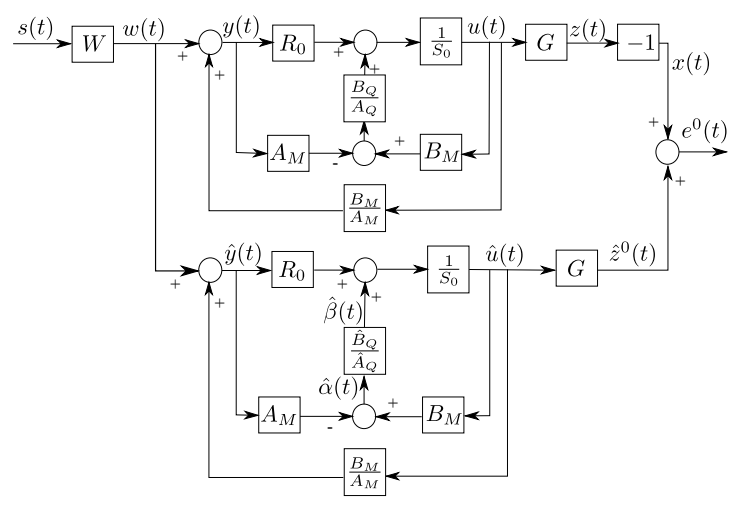

Fig. 17. Equivalent system representation

Considering a $Q\left(q^{-1}\right)$ filter as in eq. (4), the polynomial $S\left(q^{-1}\right)$ given in eq. (3) can be rewritten as

$$
S\left(q^{-1}\right)=1+q^{-1} S^{*}=1+q^{-1}\left(\left(A_{Q} S_{0}\right)^{*}-B_{Q} B_{M}^{*}\right) .
$$

Under hypothesis 3 (perfect matching condition) the output of the primary path can be expressed as

$$
x(t)=-z(t)=-G\left(q^{-1}\right) u(t)
$$

and the input to the Youla-Kucera schema as

$$
y(t+1)=w(t+1)+\frac{B_{M}}{A_{M}} u(t+1)
$$

where $u(t)$ is a dummy variable given by

$$
\begin{aligned}
u(t+1)= & -S^{*} u(t)+R y(t+1) \\
= & -\left(\left(A_{Q} S_{0}\right)^{*}-B_{Q} B_{M}^{*}\right) u(t)+\left(A_{Q} R_{0}-B_{Q} A_{M}\right) y(t+1) \\
= & -\left(A_{Q} S_{0}\right)^{*} u(t)+A_{Q} R_{0} y(t+1) \\
& +B_{Q}\left(B_{M}^{*} u(t)-A_{M} y(t+1)\right) .
\end{aligned}
$$

Similarly, the output of the adaptive feedforward filter (for a fixed $\hat{Q}$ ) is given by

$$
\begin{aligned}
\hat{u}(t+1)= & -\left(\hat{A}_{Q} S_{0}\right)^{*} \hat{u}(t)+\hat{A}_{Q} R_{0} \hat{y}(t+1) \\
& +\hat{B}_{Q}\left(B_{M}^{*} \hat{u}(t)-A_{M} \hat{y}(t+1)\right) .
\end{aligned}
$$

The output of the secondary path is

$$
\hat{z}(t)=G\left(q^{-1}\right) \hat{u}(t)
$$

Define the dummy error (for a fixed estimated set of parameters)

$$
\epsilon(t)=-u(t)+\hat{u}(t)
$$

and the residual error

$$
\left.\nu(t)=-e(t)=-(-z(t)+\hat{z}(t))=-G\left(q^{-1}\right) \epsilon(t)\right) .
$$


Equation (55) can be rewritten as

$$
\begin{aligned}
& u(t+1)=-\left(A_{Q} S_{0}\right)^{*} \hat{u}(t)+A_{Q} R_{0} \hat{y}(t+1)+B_{Q}\left(B_{M}^{*} \hat{u}(t)\right. \\
& \left.\quad-A_{M} \hat{y}(t+1)\right)-\left(A_{Q} S_{0}\right)^{*}(u(t)-\hat{u}(t))+A_{Q} R_{0}(y(t+1) \\
& \quad-\hat{y}(t+1))+B_{Q}\left[B_{M}^{*}(u(t)-\hat{u}(t))-A_{M}(y(t+1)-\hat{y}(t+1))\right] .
\end{aligned}
$$

Taking into consideration eqs. (10), (54)

$$
\begin{aligned}
B_{Q}\left[B_{M}^{*}(u(t)-\hat{u}(t))-A_{M}(y(t+1)-\hat{y}(t+1))\right] & = \\
=B_{Q}\left[B_{M}^{*} \epsilon(t)-A_{M} \frac{B_{M}^{*}}{A_{M}} \epsilon(t)\right] & =0
\end{aligned}
$$

and substracting equation (56), from (60) one obtains

$$
\begin{aligned}
\epsilon(t+1)= & -\left(\left(-A_{Q}+\hat{A}_{Q}\right) S_{0}\right)^{*} \hat{u}(t)+\left(-A_{Q}+\hat{A}_{Q}\right) R_{0} \hat{y}(t+1) \\
& +\left(-B_{Q}+\hat{B}_{Q}\right)\left[B_{M}^{*} \hat{u}(t)-A_{M} \hat{y}(t+1)\right] \\
& -\left(A_{Q} S_{0}\right)^{*} \epsilon(t)+A_{Q} R_{0} \frac{B_{M}^{*}}{A_{M}} \epsilon(t) .
\end{aligned}
$$

Passing the terms in $\epsilon(t)$ on the left hand side, one gets:

$$
\begin{aligned}
{[1+} & \left.q^{-1}\left(\frac{A_{M}\left(A_{Q} S_{0}\right)^{*}-A_{Q} R_{0} B_{M}^{*}}{A_{M}}\right)\right] \epsilon(t+1)=\frac{A_{Q} P_{0}}{A_{M}} \epsilon(t+1) \\
= & \left(-A_{Q}^{*}+\hat{A}_{Q}^{*}\right)\left[-S_{0} \hat{u}(t)+R_{0} \hat{y}(t)\right] \\
& +\left(-B_{Q}+\hat{B}_{Q}\right)\left[B_{M} \hat{u}(t+1)-A_{M} \hat{y}(t+1)\right]
\end{aligned}
$$

Using eqs. (59) and (19) one gets:

$$
\nu(t+1)=\frac{A_{M}\left(q^{-1}\right) G\left(q^{-1}\right)}{A_{Q}\left(q^{-1}\right) P_{0}\left(q^{-1}\right)}(\theta-\hat{\theta})^{T} \phi(t),
$$

which corresponds to eq. (17) and this ends the proof.

\section{APPENDIX B}

\section{PROOF OF LEMMA 5.1}

Proof: Using Theorem 3.2 from [23], under the condition (39), (34), (35) and (36) hold.

However in order to show that $\nu^{0}(t+1)$ goes to zero one has to show first that the components of the observation vector are bounded. The result (36) suggests to use the Goodwin's "bounded growth" lemma ([26] and Lemma 11.1 in [23]). Provided that one has:

$$
\begin{gathered}
\left|\psi^{T}(t) F(t) \psi(t)\right|^{\frac{1}{2}} \leq C_{1}+C_{2} \cdot \max _{0 \leq k \leq t+1}\left|\nu^{0}(k)\right| \\
0<C_{1}<\infty, \quad 0<C_{2}<\infty, \quad F(t)>0,
\end{gathered}
$$

$\|\psi(t)\|$ will be bounded. So it will be shown that (65) holds. This will be proved for algorithm I (for algorithms II and III the proof is similar).

From (14) one has

$$
-\hat{z}(t)=\nu(t)+x(t) .
$$

Since $x(t)$ is bounded (output of an asymptotically stable system with bounded input), one has

$$
\begin{aligned}
\left|\hat{u}_{f}(t)\right|= & |G \hat{u}(t)|=|\hat{z}(t)| \leq C_{3}+C_{4} \cdot \max _{0 \leq k \leq t+1}|\nu(k)| \\
& \leq C_{3}^{\prime}+C_{4}^{\prime} \cdot \max _{0 \leq k \leq t+1}\left|\nu^{0}(k)\right| \\
0 & <C_{3}, C_{4}, C_{3}^{\prime}, C_{4}^{\prime}<\infty
\end{aligned}
$$

since $|\nu(t)| \leq\left|\nu^{0}(t)\right|$ for all $t$. Filtering both sides of equation (10) by $G\left(q^{-1}\right)$ one gets in the adaptive case:

$$
\hat{y}_{f}(t)=G \cdot w(t)+\frac{B_{M}}{A_{M}} \cdot \hat{u}_{f}(t)
$$

Since $A_{G}$ and $A_{M}$ are Hurwitz polynomials and $w(t)$ is bounded, it results that

$$
\left|\hat{y}_{f}(t)\right| \leq C_{5}+C_{6} \cdot \max _{0 \leq k \leq t+1}\left|\nu^{0}(k)\right| ; \quad 0<C_{5}, C_{6}<\infty
$$

Using equations (19a), (19b), (27), (68) and (70) one can conclude that

$$
\left|\alpha_{f}(t)\right| \leq C_{7}+C_{8} \cdot \max _{0 \leq k \leq t+1}\left|\nu^{0}(k)\right|
$$

and

$$
\left|\beta_{f}(t)\right| \leq C_{9}+C_{10} \cdot \max _{0 \leq k \leq t+1}\left|\nu^{0}(k)\right|
$$

Therefore (65) holds, which implies that $\psi(t)$ is bounded and one can conclude that (38) also holds. End of the proof. 